\title{
Application-aware scheduling for VoIP in Wireless Mesh Networks
}

\author{
Nico Bayer ${ }^{\mathrm{a}, *}$, Bangnan $\mathrm{Xu}^{\mathrm{a}}$, Veselin Rakocevic ${ }^{\mathrm{b}}$, Joachim Habermann ${ }^{\mathrm{c}}$ \\ a Deutsche Telekom/T-Systems, Deutsche-Telekom-Allee 7, 64295 Darmstadt, Germany \\ ${ }^{\mathrm{b}}$ School of Engineering and Mathematical Sciences, City University London, London ECIV OHB, United Kingdom \\ ${ }^{\mathrm{c}}$ University of Applied Sciences Friedberg, Wilhelm Leuschner Straße 13, 61169 Friedberg, Germany
}

Keywords:

Wireless Mesh Network

Voice over IP - VoIP

Quality of Service - QoS

Delay

Jitter

Packet error rate

Multi-hop

Triple Play

$R$-score

\section{A B S T R A C T}

Wireless Mesh Networks (WMNs) are seen as a means to provide last mile connections in Next Generation Networks (NGNs). Because of their auto configuration capabilities and the low deployment cost WMNs are considered to be an efficient solution for the support of multiple voice, video and data services in NGNs. This paper looks at the optimal provision of resources in WMNs for Voice over IP (VoIP) traffic, which has strict performance require ments in terms of delay, jitter and packet loss. In WMNs, because of the challenges intro duced by wireless multi hop transmissions and limited resources, providing performance quality for VoIP comparable to the voice quality in the traditional circuit switched net works is a major challenge.

This paper analyses different scheduling mechanisms for TDMA based access control in mesh networks as specified in the IEEE 802.16 2004 WiMAX standard. The performance of the VoIP applications when different scheduling mechanisms are deployed is analysed on a variety of topologies using ns 2 simulation and mathematical analysis. The paper con cludes that on demand scheduling of VoIP traffic typically deployed in 802.11 based WMNs is not able to provide the required VoIP quality in realistic mesh WiMAX network scenarios and is therefore not optimal from a network operator's point of view. Instead, it is shown, that continuous scheduling is much better suited to serve VoIP traffic. The paper then proposes a new VoIP aware resource coordination scheme and shows, through simu lation, that the new scheme is scalable and provides good quality for VoIP service in a wide range of network scenarios. The results shown in the paper prove that the new scheme is resilient to increasing hop count, increasing number of simultaneous VoIP sessions and the background traffic load in the network. Compared to other resource coordination schemes the VoIP aware scheduler significantly increases the number of supported calls.

\section{Introduction}

Internet users increasingly require flexibility and mobil ity when using network services. This can be achieved using wireless access networks, which have become very popular in recent years. One viable solution for providing wireless access are Wireless Mesh Networks (WMNs). The main advantage of WMNs is the ability of wireless nodes to com

\footnotetext{
* Corresponding author. Tel.: +49 1784764263.

E-mail address: nico.bayer@telekom.de (N. Bayer).
}

municate over multiple wireless hops which increases radio coverage area, provides coverage in shadowed areas and enhances system performance. This multi hop commu nication enables network connectivity between stations that are outside of their typical single hop transmission range. It is interesting to note that multi hop communica tion is also a feature of wireless ad hoc networks where the focus is on mobility support, low power operation and end user device design. Compared to wireless ad hoc networks, WMNs are based on static infrastructure compo nents with unlimited power supply and the focus is typically on network reliability and network capacity. 
Today, the Internet is not just responsible for delivering traditional data services but also telephony services using Voice over IP (VoIP) technology and television services using television via IP (IPTV). These real time services have strict Quality of Service (QoS) requirements in terms of de lay, loss, jitter and bandwidth. It is generally regarded that WMNs are not able to meet the strong QoS requirements as these networks increase the packet delay and packet loss due to their wireless multi hop transmission. This paper shows that this is not necessarily true and analyses the VoIP quality in WMNs in detail, by measuring delay, jitter and packet loss of VoIP application in a range of scenarios.

The potential of WMNs generated a lot of activity at various levels in the networking community. Firstly, the vendors are pushing their proprietary mesh products. For example BelAir [1], Saxnet [2] and Cisco [3] are offering mesh solutions for small and large scale Internet connec tivity. Secondly, a number of community mesh networks have been implemented, providing connectivity and in creased capacity for wireless users. The MIT Roofnet [4] and the Freifunk in Berlin [5] are examples of community networks currently containing up to 200 access points and are continuously growing in size. Thirdly, research testbeds such as MagNets [6], RescueMesh [7] or MeshBed [8] have been developed to experimentally evaluate mesh networks as well as to understand their limitations and their full potential. Finally, the standardisation activities, especially within the Institute of Electrical and Electronics Engineers (IEEE) 802 Working Group, focus on multi hop mesh networks for broadband wireless access. Examples of these include the IEEE 802.11s and the IEEE 802.16 Worldwide Interoperability for Microwave Access (Wi MAX) [9] initiatives.

There are currently two main Medium Access Control (MAC) protocol concepts under consideration for WMNs. The random access protocols, such as the Carrier Sense Multiple Access with Collision Avoidance (CSMA/CA) used in the IEEE 802.11 standard [10] are not capable of meeting the "carrier grade" requirements of network operators be cause of increased network contention and thus increased delay and packet loss in highly loaded networks [11]. This is the main reason why coordinated access protocols, such as the Time Division Multiple Access (TDMA), used in IEEE 802.16 networks, are currently under consideration for Wireless Mesh Networks. The IEEE 802.16 2004 standard specifies the resource control and the reservation mecha nisms to realise QoS support for real time applications. However, the standard provides only a framework for the mesh mode and in its current state leaves a number of open issues. For example, the standard defines two different scheduling approaches, namely "centralised" and "distributed", but the exact mechanism for assigning and distributing slots for the data transmissions within the mesh network (the actual scheduling process) is not defined.

This paper adopts the mesh framework of the IEEE 802.16 2004 standard [9] and analyses the operation of TDMA based WMNs in chain and grid topologies. The pa per provides a detailed ns 2 simulation and analytical investigation of the network performance for VoIP traffic in terms of quality and scalability. The paper then intro duces scheduling mechanisms for mesh networks and gives their detailed evaluation. Two different scheduling approaches are presented: on demand and the continuous scheduling. For the continuous scheduler, two well known resource coordination schemes are considered and a new, application aware scheduling is proposed. Analytical mod els are developed for the analysis of the VoIP quality and network scalability for various TDMA configurations. To the best of our knowledge, this is the first paper proposing application aware scheduling for VoIP in TDMA based WMNs and analysing in detail the influence of scheduling on the VoIP performance in such systems.

The results and investigations in this paper, while focusing on the IEEE 802.16 2004 network standard, are general in nature and can be applied to any other TDMA based multi hop system. The rest of the paper is organised as follows. Section 2 discusses related work. Section 3 anal yses the resource scheduling in the TDMA based WMNs and describes the different scheduling approaches that are used within this paper, including the new VoIP aware scheduler. Section 4 provides an overview of the observed network scenarios and the simulator, including the simula tion parameters, the analytical models, and the use of the International Telecommunication Union (ITU) T E Model as a quality metric for the VoIP applications. Section 5 pre sents the results of the quality and scalability investiga tions. Finally, Section 6 concludes the paper.

\section{Related work}

The support for real time applications like VoIP in wire less access networks has been a major research challenge in the recent past. A substantial amount of work investi gating the support for VoIP in single hop wireless net works has been published. For example, in [12,13] the performance of VoIP in IEEE 802.16 based backhaul net works operating in the Point to Multipoint (PMP) mode is investigated. [12] evaluates the impact of the scheduling mechanism on the quality of the VoIP service as well as the network scalability for the VoIP traffic and compares the performance of three different scheduling mechanisms. The authors of [13] achieve good VoIP quality through a balance between delay and loss by exploiting and combin ing IEEE 802.16 MAC layer features such as Forward Error Correction (FEC), Automatic Repeat Request (ARQ), aggre gation and mini slot (MS) allocation. The work in [13] pro vides a detailed analysis of the performance of VoIP traffic in TDMA based networks, however does not consider the impact of multi hop communication.

VoIP in the Wireless Mesh Networks has mainly been analysed for IEEE 802.11 based networks. For example, Lee et al. [14] gives a detailed evaluation of the IEEE 802.11 network capacity for VoIP traffic. Improving the quality as well as the capacity by employing aggregation methods and header compression has been considered in [15 17]. However, due to the difference in the operation of the MAC layer, these methods cannot be directly applied to the TDMA based WMNs.

As previously mentioned, the scheduling mechanism has a significant impact on the performance and scalability 
in TDMA networks. In [ 18 22] scheduling mechanisms are proposed to improve the performance in IEEE 802.16 WMNs. While Cao et al. [18] analyses the Orthogonal Fre quency Division Multiple Access (OFDMA) and improves the sub channel assignment process, the other papers aim to improve efficiency by optimising the spatial re source reuse. The contribution of these papers is in the per formance optimisation for TDMA based WMNs, but they do not focus on real time applications like VoIP. Real time applications are considered in [23] where simulation and analysis are used to investigate the centralised scheduler of the IEEE 802.16 mesh MAC layer. The results include a trade off analysis between scalability and performance. In addition, the influence of the network configuration parameters on the scheduling delay is investigated. An other recent work in [24] presents delay studies for WMNs and proposes a topology aware scheduler which is able to decrease packet delay. However, these papers do not explicitly look at VoIP traffic and they do not evaluate other network parameters that are important for voice applications like jitter and packet error rate (PER).

\section{Scheduling in TDMA-based WMNs}

\subsection{The mesh TDMA MAC layer}

The TDMA frame structure used within this paper is based on the IEEE 802.162004 standard (see Fig. 1). The length of the TDMA frame is defined by the Frame Length $(F L)$ parameter (e.g. 4 and $10 \mathrm{~ms}$ ) and the frame is divided into the control subframe and the data subframe.

A slot in the control subframe is called a Transmission Opportunity (TO). The TOs can only be used for the trans mission of signalling messages. There are two types of con trol subframes: the network control subframe and the schedule control subframe. The network control subframe can be used either when new nodes join the mesh network, or for periodic broadcasting of network configuration mes sages. The schedule control subframe can be used for the transmission of signalling messages used for the schedul ing of the resources in the data subframe.

The slots in the data subframe are called mini slots (MSs) and they are mainly used for the transmission of the data packets. The scheduling mechanism is responsible for the allocation and reservation of MSs in the data sub frame. A series of MSs allocated to a mesh station is called a burst. A burst is used by the station to send one or more MAC packet data units (PDUs).
To coordinate the usage of the MSs in the data sub frame, the standard defines two scheduling modes: centra lised and distributed. The two modes can be implemented concurrently in the network. The centralised scheduler ap plies a two way handshake to request and grant resources in the data subframe. In particular, normal mesh nodes re quest resources from the mesh base station and the mesh base station grants TOs among all the requesting nodes. The problem with the centralised scheduling method is that it does not scale very well due to the large number of signalling messages needed every time a new mesh node joins the network [25]. Also, the centralised sched uler does not perform as well as the decentralised sched uler in networks where the coverage area of multiple mesh base stations is overlapping. This is due to the fact, that for such a scenario coordination between the mesh base stations and the centralised scheduler is required. In addition, the centralised approach reduces the mesh topol ogy to a tree topology and thus unnecessarily limits the number of routes that can be utilised [26]. For these reasons, the focus of this paper is on the distributed scheduling.

Using the distributed scheduler, the mesh nodes compete for the bandwidth within a two hop extended neighbour hood of the two communicating nodes, where the neigh bourhood includes all one hop neighbours of a given node as shown in Fig. 2. Distributed scheduling can be fur ther classified into coordinated distributed scheduling and uncoordinated distributed scheduling. The coordinated distributed scheduling allows mesh nodes to transmit dis tributed scheduling signalling (MSH DSCH) messages requesting bandwidth in the control subframe in a colli sion free manner. In uncoordinated scheduling, the MSH DSCH messages are sent in free MSs of the data subframe where they may collide. Mesh nodes are free to use either of these two methods for the distributed scheduling.

In the distributed scheduler, in order to reserve the bandwidth for the data transmission between two mesh nodes, whether for the direct communication or for the communication to the mesh base station, a three way handshake (TWHS) mechanism is used. Handshake mes sages are sent within the information elements (IEs) of MSH DSCH messages [9]. The example network shown in Fig. 2 will be used in order to explain this method. If node $A$ has data to send to node $B$, node $A$ will initiate the TWHS by sending a request (REQ) contained within the MSH DSCH Request IE [9] together with an availability (AVA) which is contained within the MSH DSCH\{Availability\} IE

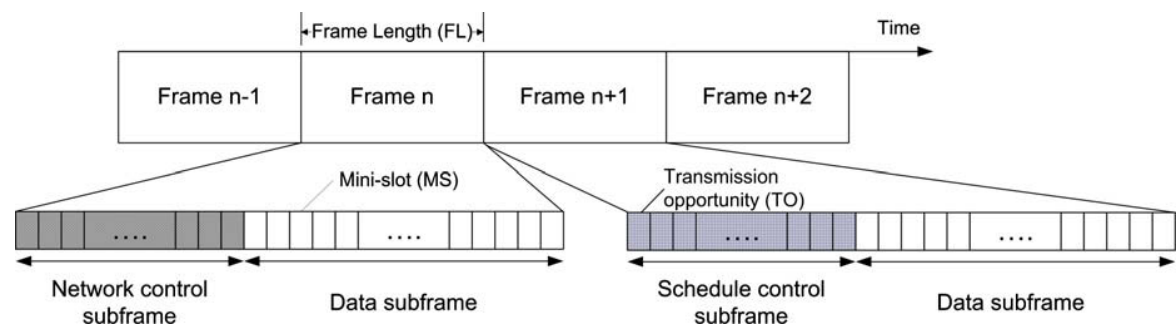

Fig. 1. TDMA frame structure. 


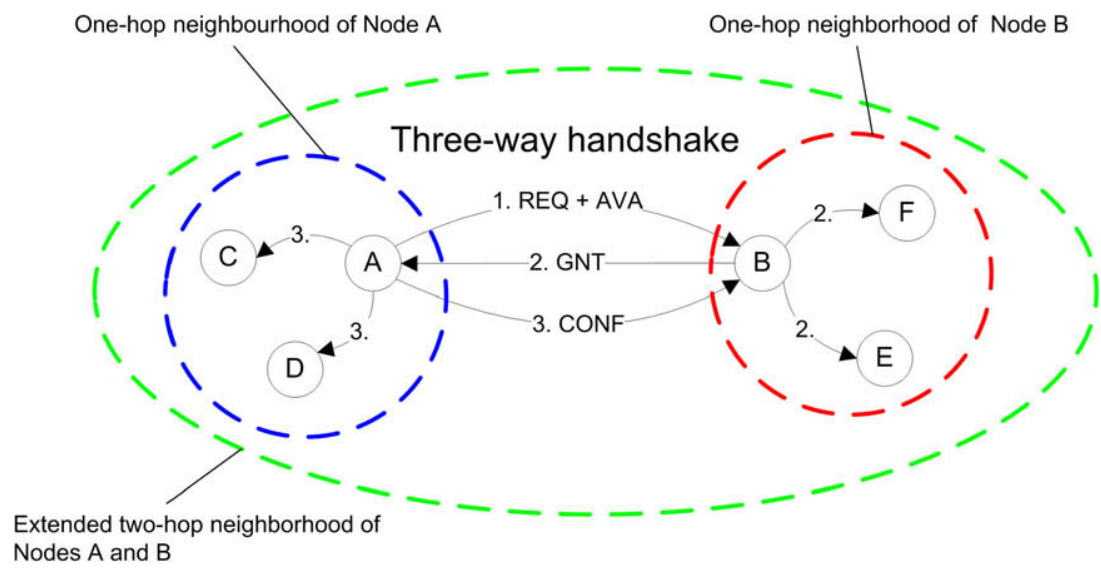

Fig. 2. Three-way handshake of the distributed scheduler.

[9] to node $B$ listing the required bandwidth and available MSs of node $A$. All one hop neighbours of node $A$, namely nodes $C$ and $D$, also receive these messages and in order to avoid that MSs are assigned multiple times, they mark these MSs as unavailable. Node $B$ will choose enough MSs from that list to fulfil the bandwidth requirement and will reply with a grant (GNT) contained within the MSH DSCH $\{$ Grant $\}$ IE [9] listing the MSs to be used for communi cation between nodes $A$ and $B$. All the nodes in the one hop neighbourhood of node $B$, namely nodes $E$ and $F$, will also receive the GNT and will mark the advertised MSs used be tween $A$ and $B$ as unavailable. Node $A$ must now send a con firmation (CONF) (which is simply a copy of the GNT) in order to confirm the receipt of the original GNT. Therefore, all the nodes in the one hop neighbourhood of node $A$, nodes $C$ and $D$, will receive the CONF and reserve those MSs. Only after node $A$ has transmitted the CONF it can begin to send data using the allocated MSs. Following the three way handshake method, the extended two hop neighbourhood is defined. The extended two hop neigh bourhood defines the area in which MSs may not be reused. However, the stations are free to reuse resources outside of this area. The mesh distributed scheduler does not define any method for assigning MSs, so MSs are free to be reused outside the extended two hop neighbourhood in which they are being used. It should be noted that the transmis sion timing of MSH DSCH messages is also a very important factor that has much influence on the network perfor mance. However, this is not considered in this paper and more details can be found in [27].

As can be seen, the TWHS mechanism defines the num ber of hops between two links which can be scheduled for concurrent transmission. This distance between these links is called the reuse distance $\alpha . \alpha$ is explained using Fig. 3 in which node $D$ wants to send a packet via link $d$ to node $E$. According to the distributed scheduler the TWHS is per formed in order to reserve the required resources for the transmission of the data packet. Because of the shared medium the REQ + AVA and the CONF transmitted by node $D$ are also received by node $C$. Hence, the links $c$ and $b$ cannot be scheduled for the transmission during the trans mission on link $d$. Nodes $B$ and $A$ are not aware of the trans mission on link $d$ as they have not received one of the signalling messages. Thus, link $a$ is the first link that can be scheduled for the transmission concurrent to the trans mission on link $d$. This holds true for link $g$ as the same explanation applies here as well. It can be seen, that the TWHS mechanism results in $\alpha=3$.

While the framework on how to request and assign bandwidth is defined by the IEEE 802.162004 standard, the exact scheduling rules and mechanisms as well as the resource coordination schemes are left undefined. Within the following sections, two different scheduling mecha nisms for different types of traffic are proposed.

\subsection{On demand scheduling}

This section describes the on demand scheduling approach that is comparable to the Distributed Coordina tion Function (DCF) of the IEEE 802.11 standard. Packets are treated on a first come first serve basis. This approach is characterised by:

- The MSs are requested on demand which means that a mesh node only requests resources in the case it has received one or more data packets. These packets can be received either from the upper layer (the node has generated the packet itself), from a neighbouring mesh node or from a client associated to the mesh node.

- To avoid the situation where one user claims all MSs, the number of MSs that can be requested in each request is limited to $M S_{\max }$.

- The number of future frames in which a node is allowed to request for MSs is limited to FrLo. This avoids wasting resources and large delays for new connections. It also simplifies the harmonisation of different scheduling pol icies and thus different traffic types.

- The granter sends a GNT only if the resources listed in the AVA are available.

- If a GNT was not received within the last cycle, the requester sends a new REQ with different resources listed in the AVA.

The message exchange procedure for the on demand scheduler is shown in Fig. 4 where the requester receives a data packet (1) and sends a REQ (2) in which it requests 


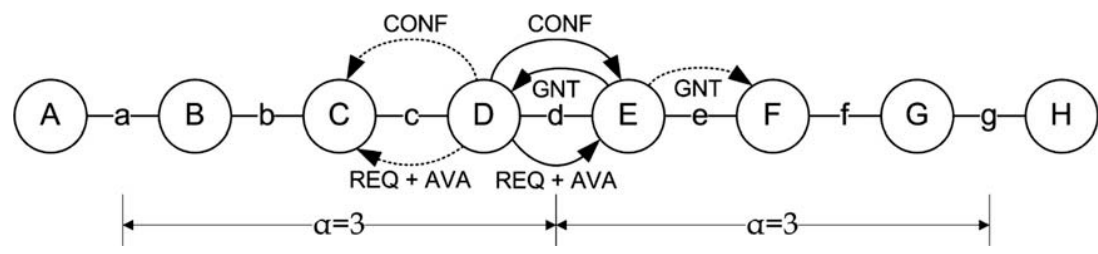

Fig. 3. Definition of the reuse-distance $\alpha$.

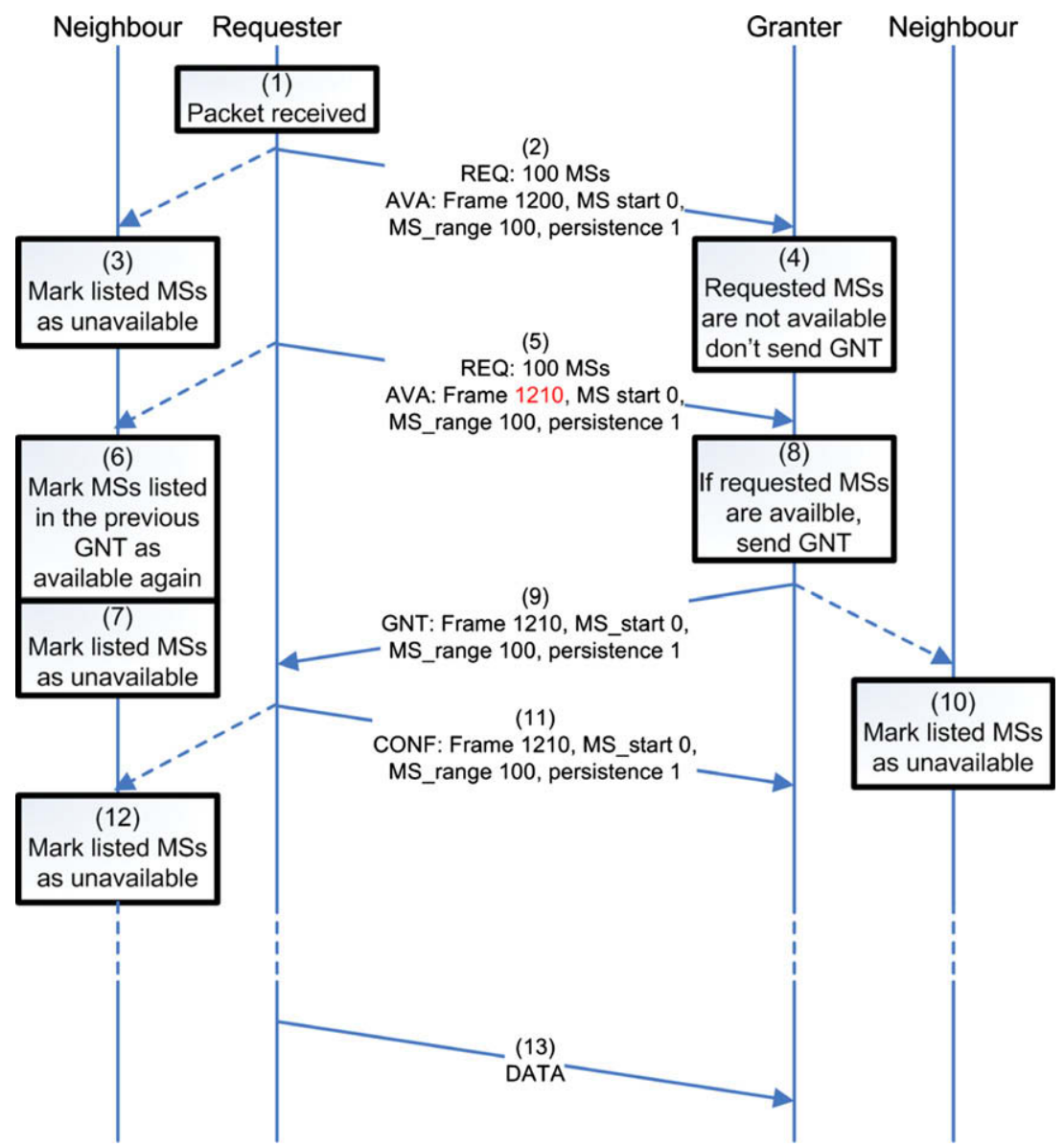

Fig. 4. Message exchange procedure for the on-demand scheduler.

100 MSs to transmit the packet. An AVA is sent along with the REQ which indicates that in frame number 1200 the slots 099 are available and can be used for transmission. A persistence of 1 indicates that this applies only for frame 1200. All nodes that receive these messages (except the granter) mark the listed resources as unavailable (3) in order to avoid that MSs are assigned twice within the extended two hop neighbourhood of the requester. In the present example, the slots listed in the availability mes sage are not free at the granter side (4). Thus, a GNT is not sent. In this case, the requester waits a specific time interval and, in the case a GNT has not been received, it sends a new REQ together with an AVA (5). However, the available MSs, listed in the AVA, are for frame 1210. All nodes that receive these messages release the resources previously marked as unavailable (from the previous re quest/availability) (6). Again the resources listed in the current availability message are marked as unavailable (7). These resources are also available at the granter (8) and a GNT is created and broadcasted (9). The GNT indi cates that the requester can use the MSs 099 in frame 1210 for transmission. Every node that receives the GNT (except the requester) marks the listed resources as unavailable (10) in order to avoid collisions. Finally, the re quester confirms with a CONF (11) (again, all direct neigh bours mark the listed resources as unavailable (12)) and transmits its data packet(s) within the assigned MSs (13).

Due to this on demand resource request, this scheduler is suited for non real time applications with bursty traffic or varying bandwidth requirements that use protocols 
such as, Hypertext Transfer Protocol (HTTP) and File Trans fer Protocol (FTP).

\subsection{Continuous scheduling}

In contrast to the on demand scheduler, the continuous scheduling approach requests resources in advance. More precisely, the resources are not requested on demand for each packet but rather for a complete session. The re sources are then reserved explicitly for the session and cannot be used for other traffic. The continuous scheduler is characterised by:

- Upon session initiation, the MSs are continuously reserved for the complete session. Thus, the MSs are periodically available and can be used to transmit data packets. Therefore, the bandwidth requirement of the session is translated into the number of MSs that are needed per frame in order to fulfil these requirements.

- The duration of the MS assignment is unlimited and if the session is finished the MSs will be made available again.
- The number of MSs that can be requested in each request is limited to avoid that one user claims all the MSs.

- Granter sends a GNT only in the case the resources listed in the AVA are available.

- If a GNT was not received within the last cycle, the requester sends a new REQ with other MSs listed in the AVA.

The message exchange procedure for the continuous scheduler is shown in Fig. 5. Its functionality is very similar to the on demand scheduler with the exception that the persistence is set to 7 which means that MSs are not only available in the listed frame but in all frames until the res ervation is cancelled. Due to these facts, step (6) in which MSs are released that have previously been marked as unavailable becomes very important. Otherwise many MSs would be marked as unavailable even if they are not used for data transmission. This would make the whole system very inefficient.

Due to this continuous resource reservation, this sched uler is well suited for real time applications with constant bandwidth requirements, i.e. VoIP.

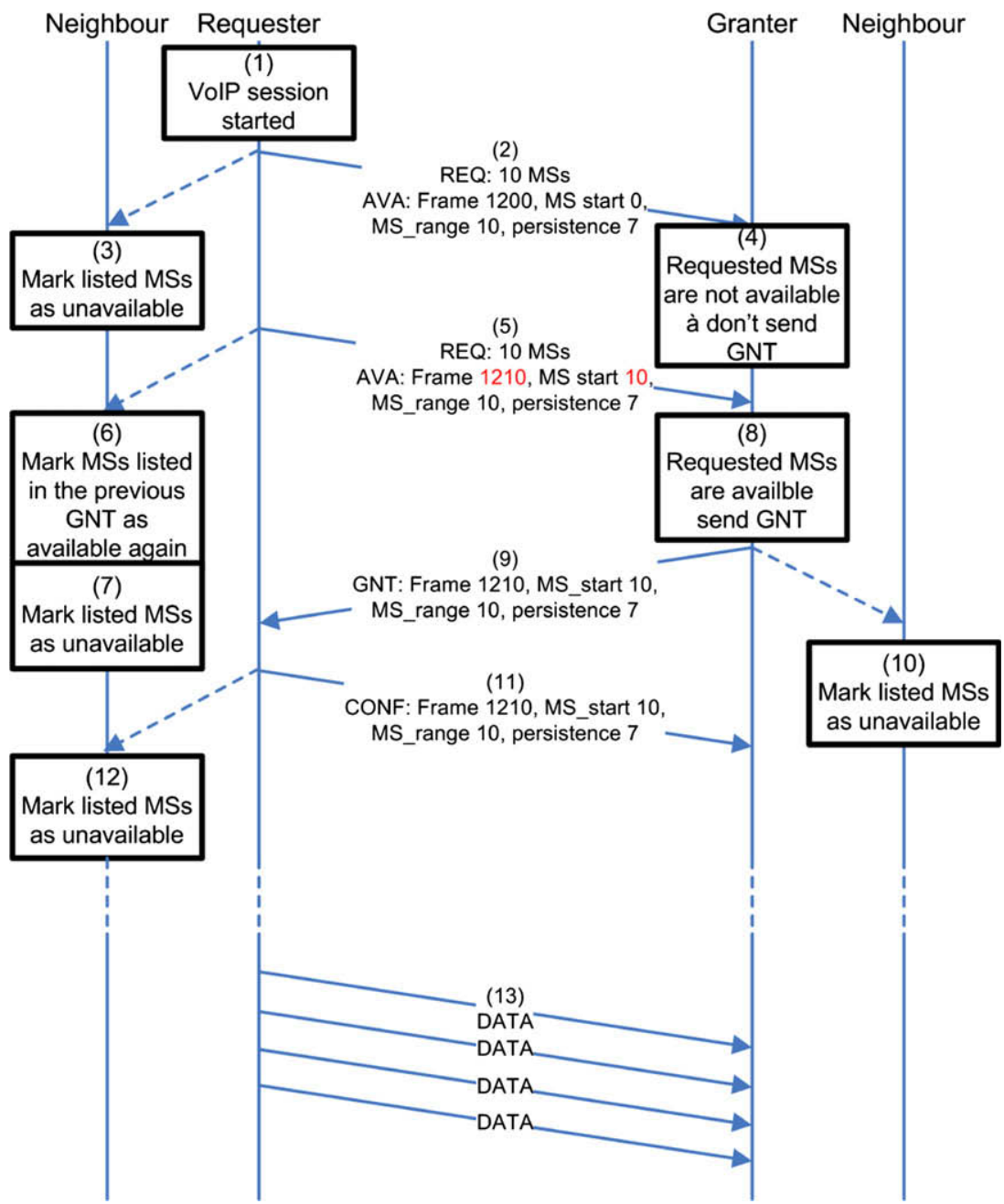

Fig. 5. Message exchange procedure for the continuous scheduler. 


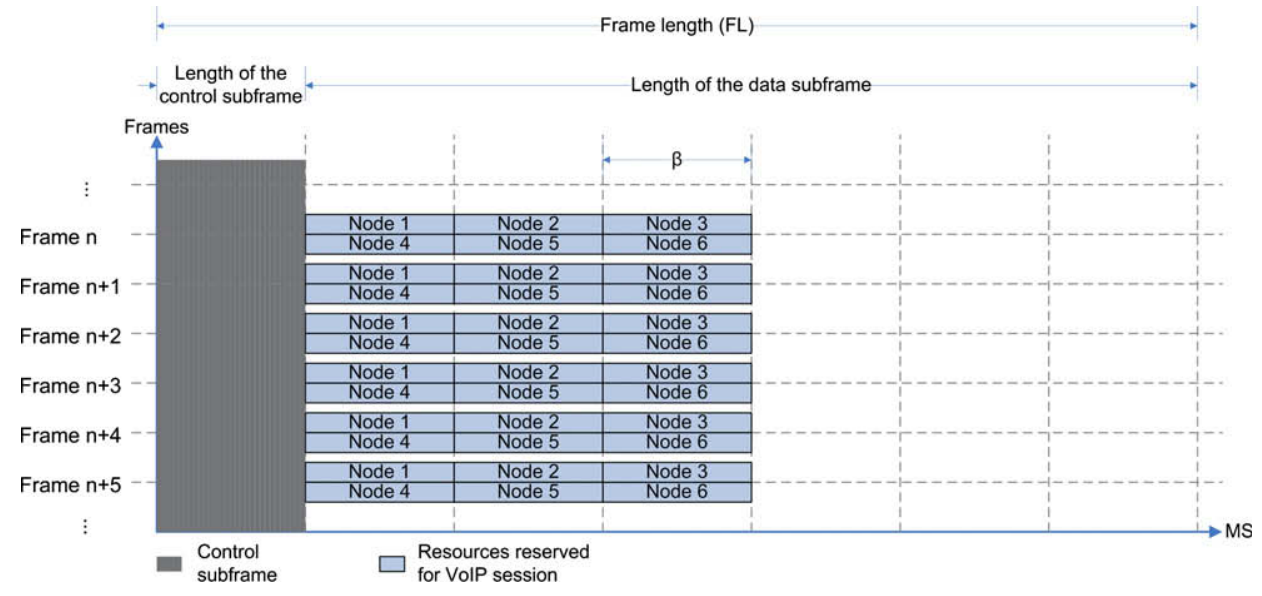

Fig. 6. Possible resource allocation for the uncoordinated resource coordination scheme based on a simple chain topology with 7 nodes.

While this mechanism describes how to request and as sign resources, it does not define how to arrange resources in a multi hop system. In the following, three resource coordination schemes are presented. The impact on the VoIP will be discussed in Section 5. The first two schemes are state of the art. However, to the best of our knowl edge, this is the first paper that investigates their influence on the VoIP traffic. Our investigation shows that these two schemes are not very efficient for delivering VoIP traffic. Therefore, we propose a new scheme called the VoIP aware resource coordination scheme.

\subsubsection{Uncoordinated resource coordination scheme}

The uncoordinated resource coordination scheme as signs MSs without considering the network topology. The aim of the scheduler is to assign the earliest available MSs in a frame. A possible resource allocation for the unco ordinated resource coordination scheme is shown in Fig. 6. It is based on a simple chain topology with 7 nodes. VoIP traffic is sent from node 1 to node 7 . In this example, nodes 1 and 4,2 and 5 and 3 and 6 are allowed to transmit simul taneously. The two reasons for this are: (1) the TWHS that permits to reuse resources within the extended two hop neighbourhood; (2) the policy of the scheduler that aims to assign the earliest available MSs in a frame.

It should be noted that in a real system normally each transmission is expanded by a guard time. This guard time is used to set the geographical distances between nodes and to allow mesh nodes to switch between receive and transmission mode and vice versa. This guard time is not taken into account in the presented simulations and calculations.

\subsubsection{Coordinated resource coordination scheme}

The coordinated resource coordination scheme is based on the idea of the topology aware scheduler as described in [24]. This scheme aims to order assigned MSs in the frame with respect to the position of the nodes in the net work. For example node $M N_{n}$, which is $n$ hops away from the destination is served before node $M N_{n} 1$ which is $n 1$ hops away. That means, that a mesh node which is further from the destination than any other mesh node is served first. A possible resource allocation is shown in Fig. 7. It is also based on the 7 node chain topology. In this example, node 1 gets the first part of the frame to transmit to node 2 . Node 2 uses the second part of the frame to transmit to node 3 and so forth.

Because of this topology aware distribution and arrangement of resources, packets can be delivered over multi hop paths within a single frame.

To realise this scheduler, the requester only needs to list the resources within the availability message which come later in the frame than the resources in which the reques ter receives the data packets to be forwarded. Hence, the requester will automatically be scheduled for transmission after it has received the data packets (if the resources are also free at the granter).

\subsubsection{VoIP aware resource coordination scheme}

To improve efficiency, this paper proposes the VoIP aware resource coordination scheme which is an enhance ment of the coordinated scheme. In contrast to the coordi nated scheme, resources are not reserved in every frame but are reserved based on the packet interval of the VoIP codec. For example, the G.711 codec creates one voice packet every $20 \mathrm{~ms}$. However, current scheduling ap proaches do not consider that fact and simply assign re sources in each frame, independent of the frame length.

For a frame length of $4 \mathrm{~ms}$ this means that, effectively, a packet will only be transmitted in every fifth frame. To overcome this inefficiency, the VoIP aware resource coor dination scheme considers the packet interval and assigns resources not in every frame, but according to the packet interval of the VoIP codec as well as the frame length. Fig. 8 shows a possible resource allocation considering a scheduling interval of four. This means, that for a VoIP ses sion, resources are reserved in every fourth frame. In this example the VoIP session is scheduled in frame $n$ and every fourth frame thereafter. Due to this codec aware schedul ing approach, the network can be used more efficiently.

Eq. (1) is used to calculate the scheduling interval $(I)$ from the TDMA frame length $(F L)$, the packet interval of 


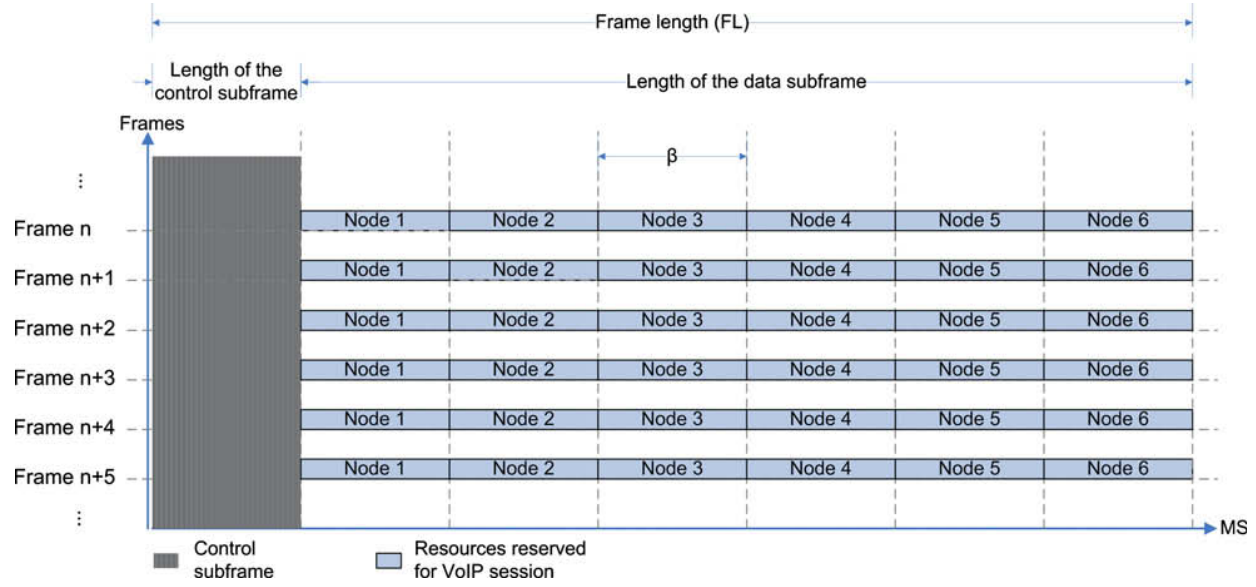

Fig. 7. Possible resource allocation for the coordinated resource coordination scheme based on a simple chain topology with 7 nodes.

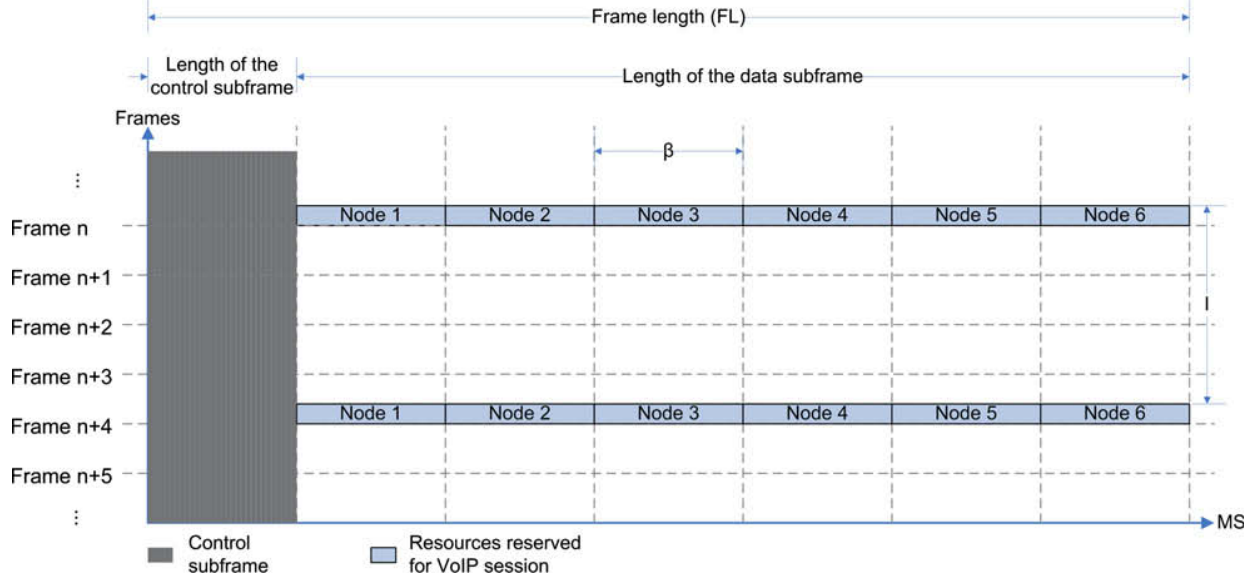

Fig. 8. Possible resource allocation for the VolP-aware resource coordination scheme based on a simple chain topology with 7 nodes.

the VoIP codec $\left(I_{V o I P}\right)$ and the desired amount of over pro visioning $(O P)$ :

$I \quad\left\lfloor\frac{I_{V O I P}}{F L} *(1 \quad 0 P)\right\rfloor$.

Some amount of over provisioning in the case packets need to be retransmitted because of packet errors or large jitter values is required. The selection of $O P$ depends upon the network operator and can be configured statically or dynamically. A dynamic approach is well suited to cope with bad channels/links. For example, in order to allow for packet re transmission, a large $O P$ value can be used in the case the channel conditions are bad and the PER is high. The analysis of appropriate $O P$ values for different channel conditions thus becomes an important issue. This is outside the scope of this paper, however an investigation is left for future work. Within this paper a static OP config uration is used.

It should also be noted that the current version of the distributed scheduler as defined in the IEEE 802.16 2004 standard does not consider the usage of a transmission interval as discussed above. It only allows resources to be assigned continuously in each frame. In order to realise the VoIP aware scheme an enhancement of the MSH DSCH\{Request\} IE, MSH DSCH\{Grant\} IE and MSH DSCH Availability\} IE is required to be able to carry infor mation about the transmission interval. This is a simple enhancement, only requiring the addition of a single field. For the focus of this paper, the size of this field must not be larger than 3 bits. With 3 bits eight different transmission interval values can be identified, which is sufficient for the traffic considered in this paper.

How each mesh node obtains $I_{V o I P}$ can be implemented in different ways and is not defined in this paper. One pos sibility is that a central control entity, which is responsible for traffic control and resource coordination (e.g. IP Multi media Subsystem (IMS) [28] and Resource and Admission Control Subsystem (RACS) [29]), informs the mesh network about this parameter. Another solution could be that each mesh node observes signalling messages between the mo bile node and the core network and obtains $I_{V o I P}$ from the Session Initiation Protocol (SIP) messages. 
Table 1

Relation between $R$-score and user satisfaction [30].

\begin{tabular}{ll}
\hline$R$-score & User satisfaction \\
\hline $100-90$ & Very satisfied \\
$89-80$ & Satisfied \\
$79-70$ & Some users dissatisfied \\
$69-60$ & Many users dissatisfied \\
$59-50$ & Nearly all users dissatisfied \\
\hline
\end{tabular}

\section{System description and evaluation environment}

\subsection{VoIP quality}

For VoIP applications the Quality of Experience (QoE) is the most important performance metric from a user's point of view. To measure the QoE, the ITU T E Model [30,31] provides an algorithm to estimate the voice quality based on different parameters and aspects of voice quality impairment. The $R$ score considers these aspects and expresses the voice quality on a scale between 0 and 100 , see Table 1 . The $R$ score is given by:

$\begin{array}{lllll}R & 100 & I_{s} & I_{d} & I_{e f}+A .\end{array}$

In this equation $I_{s}$ represents the signal to noise impair ment associated with typical switched circuit network paths. $I_{d}$ represents the influence of the mouth to ear delay of the path on the VoIP quality, $I_{e f}$ is an equipment impair ment factor that comprises impairments caused by low bit rate codecs as well as impairments due to packet loss and $A$ is the expectation factor that expresses those intangible quantities that are difficult to quantify. According to [31] as well as the default values of [30], the $R$ score can be sim plified to:

\section{$\begin{array}{llll}R & 94.2 & I_{d} & I_{e f} .\end{array}$}

The influence of delay $I_{d}$ and loss $I_{e f}$ on the VoIP quality can be analysed separately. Jitter is not directly considered in the $R$ score calculation. It is expected, that if jitter exceeds a specific threshold, packets will simply be dropped. Thus, high jitter values increase loss and will be evaluated as such in the following sections.

\subsubsection{Delay impairment}

In [31] a detailed model for the delay impairment is presented and given by:

$$
I_{d} \quad 0.024 * \delta+0.11(\delta \quad 177.3) * \operatorname{IND}(\delta \quad 177.3)
$$

$\operatorname{IND}(x)$ is an indicator function for which: $\operatorname{IND}(x)=0$ if $x<0$; otherwise $I N D(x)=1$. $\delta$ generally comprises fixed delays introduced by the application itself $\left(\delta_{\text {app }}\right)$ as well as vari able parts caused by the network $\left(\delta_{\text {netw }}\right)$ :

$\delta \quad \delta_{a p p}+\delta_{\text {netw }}$.

The application delay has a fixed value and consists of the en/decoding ( $\left.\delta_{\text {coding }}\right)$, jitter buffer $\left(\delta_{\text {jitter }}\right)$ and processing ( $\delta_{\text {proc app }}$ ) delay and is introduced by the communication endpoints. More details can be found in [31]:

$\delta_{a p p} \quad \delta_{\text {coding }}+\delta_{j i t t e r}+\delta_{\text {proc_app }}$.
Assuming a G.711 voice codec with $\delta_{\text {coding }}=20 \mathrm{~ms}$, $\delta_{\text {jitter }}=40 \mathrm{~ms}$ and a negligible value for $\delta_{\text {proc app }}$, the delay impairment on voice quality can be expressed by:

$I_{d}\left\{\begin{array}{l}0.024\left(60+\delta_{\text {netw }}\right) \\ \delta_{\text {netw }}<117.3 \mathrm{~ms}, \\ 0.024\left(60+\delta_{\text {netw }}\right)+0.11\left(\left(60+\delta_{\text {netw }}\right)\right. \\ \delta_{\text {netw }} \geqslant 117.3 \mathrm{~ms} .\end{array}\right.$

The network delay is dependent upon the networks through which the voice traffic is sent. An analytical model to determine the network delay in TDMA based WMNs will be discussed in Section 4.2.1 while results are pre sented in Section 5.

\subsubsection{Loss and jitter impairment}

During a voice conversation, packet loss may lead to a word being unrecognisable and this single word may hap pen to be the key word which makes the listener misunder stand the whole sentence. The sources of loss are various, especially in wireless networks. Packet loss is caused, for example, by bad channel conditions, interference, collisions as well as packet drops due to overloaded nodes.

For VoIP applications, the sending node sends packets with a constant interval. Congested networks or transmis sion errors disturb this continuous stream. Thus, delay be tween packets can vary instead of remaining constant. As the human ear is highly sensitive to jitter, jitter buffers are introduced on endpoints. These buffers buffer VoIP packets and then play them out in a steady stream. It is as sumed, that if the jitter values do not exceed the size of the jitter buffer, variations can be compensated and voice quality is not degraded. For the G.711 codec the standard buffer size is $40 \mathrm{~ms}$ ( 2 packets). However, if jitter exceeds the size of the jitter buffer, meaning that a packet is re ceived with a significantly larger delay compared to the reference packet, it will be dropped as the contained infor mation is already outdated. Thereby jitter also contributes to packet loss.

According to [31] the loss impairment of voice quality can be expressed by:

$I_{\text {ef }} \quad \gamma_{1}+\gamma_{2} * \ln \left(1+\gamma_{3} * e\right)$.

Here, $\gamma_{1}$ is the quality impairment due to coding, $\gamma_{2}$ and $\gamma_{3}$ are codec specific parameters that describe the impact of loss on the perceived voice quality and $e$ is the total packet loss probability which can be modelled by:

e $e_{\text {trans }}+\left(\begin{array}{ll}1 & e_{\text {trans }}\end{array}\right) e_{j i t t e r}$.

$e_{\text {trans }}$ is the PER of the network through which the VoIP traffic is sent and $e_{j i t t e r}$ is loss due to jitter. Assuming a G.711 voice codec with the codec specific parameters $\gamma_{1}=0, \gamma_{2}=30$ and $\gamma_{3}=15$ (taken from [13]), the loss impairment can be expressed by:

$I_{\text {ef }} \quad 30 * \ln \left(1+15 *\left(e_{\text {trans }}+\left(1 \quad e_{\text {trans }}\right) e_{j i t t e r}\right)\right)$.

For the evaluation presented in this paper $e_{\text {trans }}$ and $e_{j i t t e r}$ are determined by analysing the trace files of the simula tor. For $e_{j i t t e r}$ it is assumed that packet $i$ is dropped by the jitter buffer if $\delta_{\text {netw }} \quad \delta_{\text {netw }_{b+1}}>b g$. Hence, $e_{j i t t e r}$ is simply: 
$e_{j i t t e r} \sum_{i}^{N} P\left\{\delta_{\text {netw }} \quad \delta_{\text {netw }_{b+1}}>b g\right\}$.

Here, $\delta_{\text {netw }}$ is the delay of the reference packet and $b g$ is the length of the jitter buffer. It is obvious that the maximum jit ter of a system is an important parameter. Models for the maximum jitter estimation in TDMA based WMNs using the proposed scheduling mechanism will be discussed in Section 4.2.2 while results are presented in Section 5.

\subsection{Delay and jitter estimation}

This section describes models to calculate delay and jit ter in TDMA based WMNs.

\subsubsection{Network delay}

In Section 4.1.1 it has already been shown that the net work delay $\left(\delta_{\text {netw }}\right)$ is a critical factor that may decrease the quality of VoIP. $\delta_{\text {netw }}$ depends upon the networks through which the voice traffic is sent and in general comprises queuing $\left(\delta_{\text {queuing }}\right)$, propagation $\left(\delta_{\text {prop }}\right)$, transmission $\left(\delta_{\text {trans }}\right)$ and processing $\left(\delta_{\text {proc net }}\right)$ delay:

$\delta_{\text {netw }} \delta_{\text {queuing }}+\delta_{\text {prop }}+\delta_{\text {trans }}+\delta_{\text {proc_net }}$.

Depending on the load of these networks and other factors, $\delta_{\text {netw }}$ is not static but may vary. $\delta_{\text {queuing }}$ represents the time a packet has to wait in the queue until it can be transmit ted over the wireless link. $\delta_{\text {prop }}$ is the amount of time it takes for a signal to travel via the wireless link to the next hop. $\delta_{\text {trans }}$ takes into consideration the time needed to transmit the packet over the air and depends on the mod ulation coding scheme (MCS) that is used. Finally, $\delta_{\text {proc net }}$ is the time that is needed to process the packet at each hop.

The work presented in this paper is based on a TDMA MAC layer and the granularity of delay calculations is one Orthogonal Frequency Division Multiplex (OFDM) symbol. It is assumed that the scheduler assigns resources only after a packet has been received and processed successfully. Therefore, $\delta_{\text {proc net }}$ can be neglected. It is also assumed that packet reception is finished after the end of an OFDM symbol. Therefore, $\delta_{\text {prop }}$ can also be neglected. Considering the assumptions above as well as the charac teristic of multi hop mesh networks, $\delta_{\text {netw }}$ can be calcu lated using Eq. (13) in which $N$ represents the number of hops in the network:

$\delta_{\text {netwWMN }} \sum_{i}^{N}\left(\delta_{\text {queuing }_{i}}+\delta_{\text {trans }_{i}}\right)$.

Since the scheduling algorithm and resource coordination scheme have large impact on the packet delay, analytical models for determining $\delta_{\text {netw }}$ for the three resource coordi nation schemes of the continuous scheduler are presented here. These models assume that the MCS is static and equal for all links within the WMN.

Regarding the continuous scheduler and the uncoordi nated resource coordination scheme, the average network delay can be expressed as:

$\overline{\delta_{\text {netwWMN }} \text { uncoord }} \quad\left\lfloor\frac{H C}{\alpha}\right\rfloor *\left(\begin{array}{ll}F L & \left.\alpha * \beta * T_{S}\right)+\beta * H C * T_{s}+\frac{F L}{2} .\end{array}\right.$
Eq. (14) has been developed through consideration and de tailed analysis of the slot based channel access mechanism. The first term in this equation accounts for the fact that the resource assignment is not coordinated. It consists of the number of hops $(H C)$, the reuse distance $(\alpha)$ (see Section 3.1 ), the number of OFDM symbols required to transmit a packet ( $\beta$, which considers the packet size, MAC overhead, preambles, etc.) and the OFDM symbol duration $\left(T_{s}\right)$. This first term is motivated by the fact that nodes always aim to assign the earliest resources in a frame. Therefore, after the reuse distance resources can be reused with the effect that packets have to wait until the next frame to be trans mitted. The second term represents the transmission delay and depends on the MCS that is used and thus, the number of OFDM symbols a VoIP packet needs to be transmitted over every hop $(\beta)$. The third term is the average time a packet has to wait until it can be transmitted. As the frame length is not exactly a multiple of the packet interval but something above or below that value, the time between the arrival of a VoIP packet and the transmission of this packet constantly varies. For example, assume that packet $n$ arrives directly before the transmission slots, but packet $n+1$ arrives directly after the transmission slots due to time drifts. In the first case, the queuing delay is almost zero while in the second case the queueing delay is almost equal to the frame length. Extensive simulations have shown that delay is uniformly distributed between these two extreme cases. Thus, the average queuing delay can be considered to be half the frame length.

In contrast to the uncoordinated resource coordination scheme, the coordinated scheme does not assign the earli est possible resources in a frame but assigns them depend ing on the topology of the network. Thus, the first term of Eq. (14) does not apply for the coordinated scheme and network delay is calculated according to Eq. (15):

$\overline{\delta_{\text {netwWMN }}} \quad \beta * H C * T_{s}+\frac{F L}{2}$.

Finally, the VoIP aware resource coordination scheme does not assign resources in every frame but according to the packet interval $(I)$ and the frame length $(F L) . \delta_{\text {queuing }}$ is not half the frame length but half the scheduling interval and is calculated according to:

$\overline{\delta_{\text {netwWMN }}} \quad \beta * H C * T_{s}+\frac{F L * I}{2}$.

\subsubsection{Delay jitter}

It has already been stated in Section 4.1.2 that jitter $(J)$ contributes to packet loss when jitter values are very high. It is assumed that only late packets contribute to the jitter loss but early packets do not as the buffer implementation will compensate for early packets. It should be noted that not all implementations are able to dynamically allocate additional buffers for early packets. It is assumed that such a dynamic buffer management is used. Thus, jitter loss is simply the probability that jitter is larger than the size of the jitter buffer as described in Section 4.1.2.

As previously shown in Section 4.1.2 the jitter of packet $n$ is the delay difference compared to the reference packet. Analytical models will now be presented to calculate the 
maximum jitter that a VoIP session will recognise for the different resource coordination schemes of the continuous scheduler.

For the uncoordinated and the coordinated schemes, jit ter is equal and the following equation has been developed through detailed investigation of the slot based access mechanism to determine the maximum expected jitter.

$J_{\text {uncoord }}^{\max } J_{\text {coord }}^{\max } \quad F L$.

The maximum expected jitter for the VoIP aware scheme looks very similar but also depends on $I$ and can be deter mined by:

$J_{\text {voip }}^{\max } \quad I * F L$.

\subsection{Simulation model}

\subsubsection{Simulator}

The network simulator ns 2 [32] was used to investi gate the concepts presented in this paper. For the purpose of this work, the network simulator has been enhanced.

First, the network interface layer (NetIF) has been im proved. The NetIF module represents the network interface and is responsible for the reception of packets. The current implementation in ns 2 is very basic. For a given Radio Propagation Model (RPM) the distance between the source and destination is taken in order to determine whether or not a packet is received correctly. Interference is currently not considered. However, interference due to spatial reuse is a very important issue in WMNs. This limitation forces an enhancement of ns 2 for more realistic simulations. The enhancements consider the Signal to Interference plus Noise Ratio (SINR) calculation during packet recep tion. SINR represents the ratio of the received carrier signal power $P_{r}$ in Watts to the interference power perceived during packet reception $P_{I}$ in Watts. $P_{I}$ is a function of the number of interferers $(L)$, the interference power of each interferer perceived by the receiver $\left(P_{i}\right)$ in Watts and the noise power $\left(P_{N}\right)$ in Watts. Thus, SINR in $\mathrm{dB}$ can be expressed by:

SINR $10 \log _{10}\left(\frac{P_{r}}{P_{I}}\right) \quad 10 \log _{10}\left(\frac{P_{r}}{\sum_{i{ }_{1} P_{i}+P_{N}}^{L}}\right)$.

Depending on the SINR calculated during packet reception as well as the reference measurement presented in [33], the PER can then be calculated for each packet.

Second, a new RPM has been implemented. An appro priate channel model is needed in order to obtain realistic simulation results that can be projected to real environ ments. For this paper the path loss model developed by the IEEE 802.16 Working Group (WG) [34] has been used. This model is based on measurements obtained by AT\&T wireless services across the United States at $1.9 \mathrm{GHz}$ with a receiver antenna height of $2 \mathrm{~m}$ [35] and provides three different terrain types A, B and C. Terrain A is a hilly terrain with moderate to heavy foliage density. Terrain B is either a flat terrain with moderate to heavy foliage density or a hilly terrain with light foliage density. Terrain $C$ is a flat terrain with light foliage density.

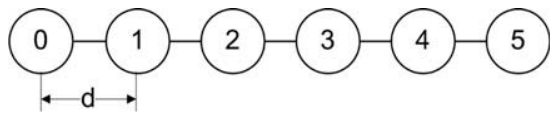

Fig. 9. Chain topology.

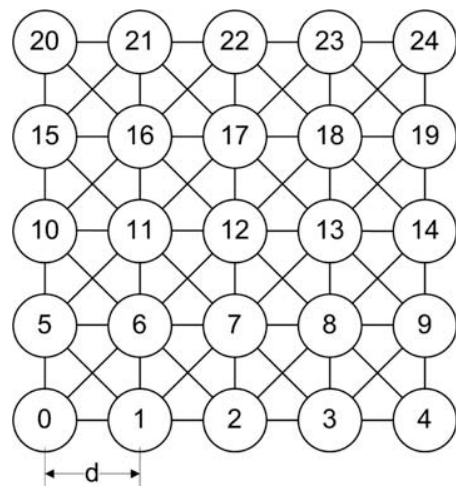

Fig. 10. Grid topology.

Third, a custom IEEE 802.16 mesh MAC module has been developed. This module includes the TDMA frame structure discussed in Section 3.1 as well as the different scheduling mechanisms described in Sections 3.2 and 3.3.

\subsubsection{Scenarios}

To understand the basic characteristics of VoIP trans mission in TDMA based mesh networks a simple 6 node chain topology was selected (see Fig. 9). This chain topol ogy allows an analysis of the basic characteristics of the different scheduling approaches. Due to the simple hop by hop packet forwarding without any cross traffic, it is possible to isolate the influences of the schedulers from any other effects in the network. In addition, the chain topology is used to validate the simulation model and the analytical model. For this purpose, in Section 5.1.1, a comparison of simulation results and analytical results for the chain topology are discussed.

Besides the analysis of the basic characteristics of the different schedulers, performance in more realistic scenar ios has also been evaluated. For this purpose, a $5 \times 5$ grid topology is used (see Fig. 10). This network topology has been recently proven to be very suitable for a real deploy ment of a WMN [36].

A random topology (e.g. random node positions, fading and random packet errors) has not been considered for several reasons. First, random topologies are not expected in a carrier grade WMN due to a planning phase in advance of the network deployment. Second, the introduction of irregular aspects would make the exact interpretation of the results very difficult as it might not be possible to iso late the influences caused by the scheduling mechanism.

\subsubsection{Simulation parameters}

Parameters used for simulations are listed in Table 2. The G.711 codec was selected for voice traffic as it is the most popular and widely deployed codec. Further details of this codec can also be found in Table 2 . 
Table 2

Simulations parameters.

\begin{tabular}{|c|c|}
\hline Parameter & Value \\
\hline RPM: model/terrain $/ a / b / c / \sigma_{s}$ & $\begin{array}{l}\text { IEEE SUI/A/4.6/0.0075/12.6/ } \\
0\end{array}$ \\
\hline TDMA: $F L / \# M S / N_{\text {SymMs }}$ & $4 \mathrm{~ms} / 110 / 1$ \\
\hline Control subframe fraction & $\approx 33 \%$ \\
\hline$d$ & $230 \mathrm{~m}$ \\
\hline MCS used & QPSK_1/2 \\
\hline$P_{N}$ & $-100 \mathrm{dBm}$ \\
\hline Antenna: type/height/gain & Omni $/ 8 \mathrm{~m} / 0 \mathrm{dBi}$ \\
\hline Queue: discipline/length & $\mathrm{FIFO} / \infty$ \\
\hline Phy: transm. power/frequ. (f)/channel BW & $35 \mathrm{dBm} / 3.5 \mathrm{GHz} / 10 \mathrm{MHz}$ \\
\hline $\begin{array}{l}\text { VoIP: codec/packet size/packet interval } \\
(\Delta)\end{array}$ & G.711/160 byte/20 ms \\
\hline FTP packet size & 500 byte \\
\hline$R_{T h r}$ & 90 \\
\hline Scheduler: $M S_{\max } / F r L o / O P / z / \beta$ & $\# S / 20 / 20 \% / 0.2 * \# S /$ \\
\hline Number of iterations chain/grid & $10 / 20$ \\
\hline
\end{tabular}

It should be noted, that the Stanford University Interim (SUI) terrain type A has been selected but without shadow ing $\left(\sigma_{s}=0\right)$. Also the MCS is fixed for all nodes to QPSK_1/2. This is in order to avoid the additional effects caused by shadowing and the MCS selection mechanism as this would make it difficult to correctly interpret the character istics of the different schedulers and their influence on the traffic performance.

\subsection{Performance evaluation metrics}

This section describes the various metrics that have been used to predict the performance of the different scheduling mechanisms.

\subsection{1. $R$ score}

The average $R$ score evaluation metric $(\bar{R})$ as described in Section 4.1 is used throughout this chapter as a measure for the VoIP quality. Eq. (20) can be used to compute the average $R$ score in the network:

$\overline{R_{n e t w}} \quad \frac{\sum_{j 1}^{Z} R_{j}}{Z}$.

Here, $Z$ is the number of VoIP streams throughout the net work and $R_{j}$ is the quality of stream $j$. Reporting the aver age $R$ score is not enough as it can occur, that the average quality is good but that the deviation from the average quality is high. Therefore, some streams will per ceive a very high quality while some will perceive very poor quality. Hence, the standard deviation of the $R$ score $\left(S_{R_{\text {netw }}}\right)$ is used and calculated by:

$S_{R_{\text {netw }}} \quad \sqrt{\frac{1}{Z}\left(\sum_{i}^{Z} R_{i}^{2}\right) \quad{\overline{R_{n e t w}}}^{2} .}$

\subsubsection{Data packet delay}

The average data packet network delay $\left(\overline{\delta_{\text {netwWMN }}}\right)$ eval uation metric is used to analyse the network delay intro duced by the different schedulers. The average network delay perceived by each node is defined as: $\overline{\delta_{\text {netwWMN }}} \quad \frac{\sum_{i{ }_{1} \delta_{\text {netwWMN }}, i}^{r} .}{r}$.

Here, $r$ is the number of received data packets by node $j$. Eq. (23) is then used to compute the average data packet delay across all nodes in the network that have at least received one data packet $(P)$ :

$\overline{\delta_{\text {netwWMN }} \text { netw }} \quad \frac{\sum_{j 1}^{P} \overline{\delta_{\text {netwWMN }}}}{P}$.

Finally, the standard deviation of delay $\left(s_{\delta_{\text {netwWmN netw }}}\right)$ can be calculated by:

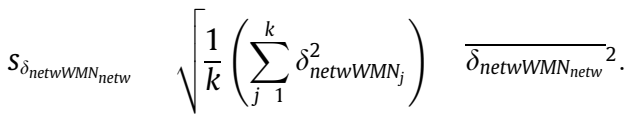

Here, $k$ is the number of data packets in the network that have successfully been received by the destination node.

\subsubsection{Data packet jitter}

The maximum data packet jitter evaluation metric is used to analyse the network delay jitter introduced by the different schedulers. The maximum data packet jitter in the whole network $\left(J_{\text {netw }}^{\max }\right)$ is defined as:

$J_{\text {netw }}^{\max } \max \left\{J_{2}, \ldots, J_{k}\right\}$.

Here, $k$ is the number of data packets in the network that have successfully been received by the destination node.

\subsubsection{Packet error rate}

The average PER $(\bar{e})$ evaluation metric can be seen as a meter for the PER as perceived by an application. The aver age PER per node is defined as follows:

$\overline{e_{j}} \quad \frac{\sum_{i}^{u} e_{j, i}}{u}$.

Here, $u$ is the number of sessions received by node $j$ and $e_{j, i}$ is the PER of session $i$ received by node $j$. Eq. (27) is then used to compute the average PER across all nodes in the network that have at least received one data session $(R)$ :

$\overline{e_{\text {netw }}} \quad \frac{\sum_{j 1}^{R} \overline{e_{j}}}{R}$.

\subsubsection{Aggregated Transmission Control Protocol (TCP) throughput}

For TCP based applications like web browsing or file transfer the throughput is the most interesting metric from a user point of view. In this paper the average aggregate TCP throughput is used as a performance metric, which is simply the sum of the throughput of every single TCP stream going through node $j$. In the relevant scenarios, node $j$ is always a mesh base station. Therefore, the average aggregated throughput metric describes the amount of traffic going to/coming from the Internet:

$\overline{G_{j}^{\prime}} \quad \sum_{i=1}^{u} G_{j, i}^{\prime}$.

Here, $u$ is the number of sessions received by node $j$ and $G_{j, i}^{\prime}$ is the average throughput of session $i$ over that complete session duration. 


\section{Performance evaluation}

In this section, the performance and quality of VoIP in TDMA based WMNs is investigated. The on demand scheduling mechanism is compared with the continuous scheduling mechanism and the different resource coordi nation schemes. Particular attention is paid to the influ ence on quality and scalability. First, the results for the chain topology are discussed followed by the results for the grid topology.

\subsection{Chain network topology}

To gain insight into the basic characteristics of VoIP transmission in TDMA based WMNs this section discusses the results for the chain topology. The chain topology allows the effects of the scheduling mechanisms to be isolated from other effects in the network. In addition, this section compares simulation results with the analytical re sults. It is shown that they are very close to each other for simulations with only one active VoIP session in which transmission errors due to interference do not exist. As the simulation model has been developed independently of the analytical models, this can be seen as a validation for both models. Due to this validation it is expected that the simulator will also provide reliable results for the more complicated grid topology discussed in Section 5.2.

\subsubsection{Quality investigations}

In this section the quality of a single VolP stream is evaluated vs. the number of hops between source and des tination for the chain scenario (Fig. 9). The aim of these re sults is to shed light onto the basic characteristics of the different schedulers. Therefore, only VoIP sessions are sim ulated without any further FTP or HTTP background traffic. The sink of the VoIP stream is always set to node 0 while the source varies in order to investigate the influence of the hop count on the VolP quality. For example, node 1 is selected for 1 hop simulations and node 5 for 5 hop simu lations. The simulation time is set to $100 \mathrm{~s}$. The VolP ses sion is started at $t=(10+R N D) \mathrm{s}$, where $R N D$ is a uniformly distributed random value between 0 and 1 .

5.1.1.1. Delay results. Fig. 11b displays average delay $\left(\overline{\delta_{\text {netwWMN }} \text { netw }}\right)$ vs. hop count $(H C)$ and compares simulation results with the analytical results obtained through Eqs. (14) (16).

Regarding the on demand scheduler, it can be seen that delay increases linearly with hop count. With every addi tional hop, delay increases by approximately $12 \mathrm{~ms}$. Of course, the smallest delay of $7.7 \mathrm{~ms}$ can be found for $H C=1$, while for $H C=5$ the average delay is $54.8 \mathrm{~ms}$. These results are expected since the TWHS needs to be performed for every single VoIP packet and for every single link. Also, the delay standard deviation $\left(s_{\delta_{\text {neerwwn nerw }}}\right.$, not shown) in creases linearly with $H C$ from $2.9 \mathrm{~ms}$ for $H C=1$ to $8.8 \mathrm{~ms}$ for $H C=5$. As delay is directly related to the quality of VoIP, this scheduler cannot provide equal VoIP quality as nodes that are close to the mesh base station will perceive lower delay and thus better VoIP quality compared to nodes several hops away from the mesh base station. However,

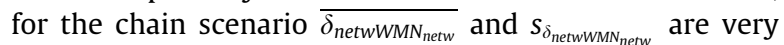
small and do not cause a significant quality degradation. In Section 5.2 it will be shown that this changes for a more realistic network topology like the grid topology.

Regarding the different resource assignment schemes of the continuous scheduler, it is obvious that all will cause much smaller delay compared to the on demand sched uler, especially if $H C$ is large. In all cases they are able to keep delay at a very low level. Furthermore, simulation and analytical results match well. Some small differences exist between simulation and analytical results which is shown for the network delay of the VoIP aware resource coordination scheme in Fig. 11d. These differences are due to the fact that the equations do not cover the system in as much detail as the simulator.

For the uncoordinated resource coordination scheme, average delay varies from approximately $2.2 \mathrm{~ms}$ in the 1 hop scenario up to approximately $6.5 \mathrm{~ms}$ in the 5 hop sce nario. It is obvious that this resource coordination scheme is also not able to provide equal quality as it causes delay to increase over distance. The delay step from the 3 hop to the 4 hop scenario is caused by the fact that resource assignment is not coordinated and that the scheduler al ways aims to assign the earliest slots that are available in the frame. This means, that for an unloaded network with out background traffic and the 4 hop scenario, that nodes 1 , 2 and 3 are not allowed to transmit (due to the TWHS) in the same MSs in order to avoid collisions. Thus, node 1 will use the first slots in the frame, node 2 will use the slots after node 1 and node 3 will use the slots after node 2 . Since the distance between node 4 and node 1 is large enough, slots used by node 1 (which are the first slots in the frame) are reused by node 4 . Due to this assignment, packets received by node 4 cannot be retransmitted immediately but have to wait until the next frame. This adds an additional delay of approximately the length of the TDMA frame.

The coordinated resource coordination scheme elimi nates the delay step of the continuous scheduler as it as signs slots based on the position of the nodes. Thus, packets can be retransmitted immediately after they have been received. In contrast to the on demand scheduler and the uncoordinated resource coordination scheme, equal quality can be provided as delay is independent of the number of hops and a delay of less than $3.3 \mathrm{~ms}$ can be pro vided independent of the location of the user.

Finally, the delay curve of the VoIP aware resource coordination scheme is shifted upwards by approximately $6 \mathrm{~ms}$ compared to the coordinated resource coordination scheme. As resources are not available in every frame, but rather according to the scheduling interval (here every fourth frame), the average time from the creation of a packet until it can be transmitted is larger. Therefore, the queuing delay is larger. However, this increased delay comes with the advantage of increased scalability which will be further discussed in Section 5.1.2. Nevertheless, in all cases the delay is kept at a very low level, independent of HC. Furthermore, it was found that, for all resource coor dination schemes of the continuous scheduler, delay is very predictable as the delay standard deviation is inde 


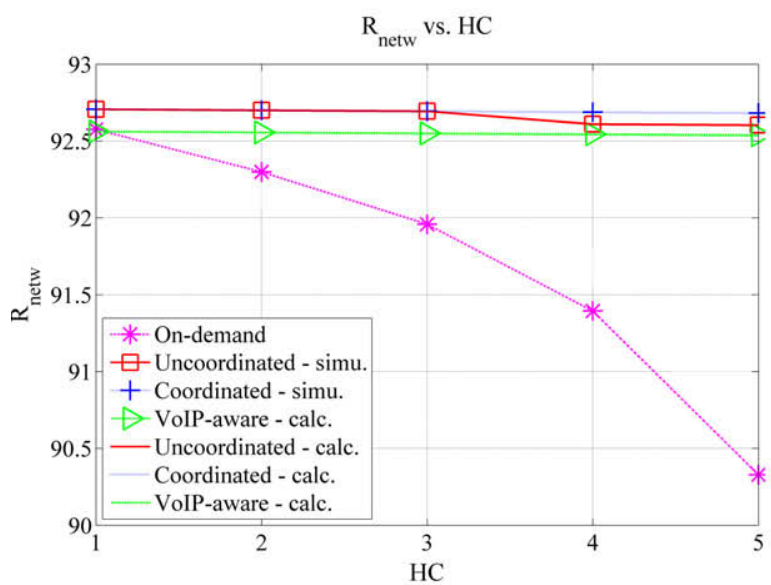

(a) Average VoIP R-score $\left(\overline{R_{\text {netw }}}\right)$

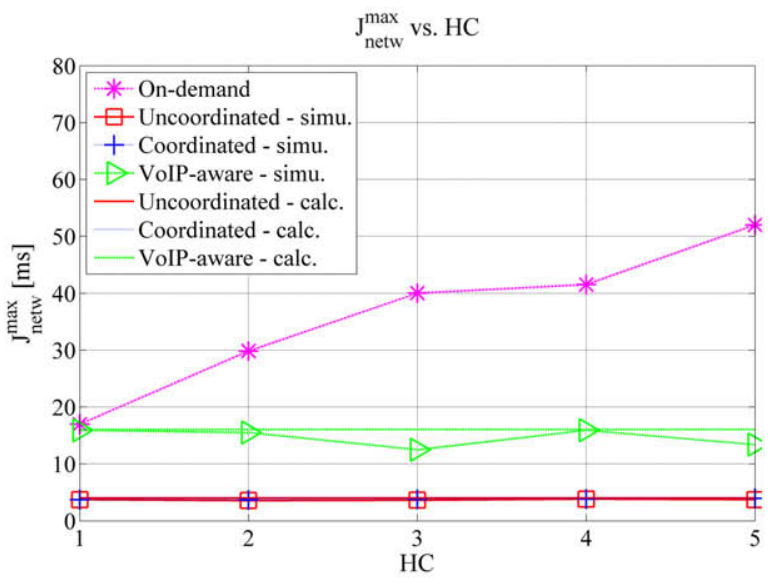

(c) Max. VoIP packet jitter $\left(J_{\text {netw }}^{\max }\right)$
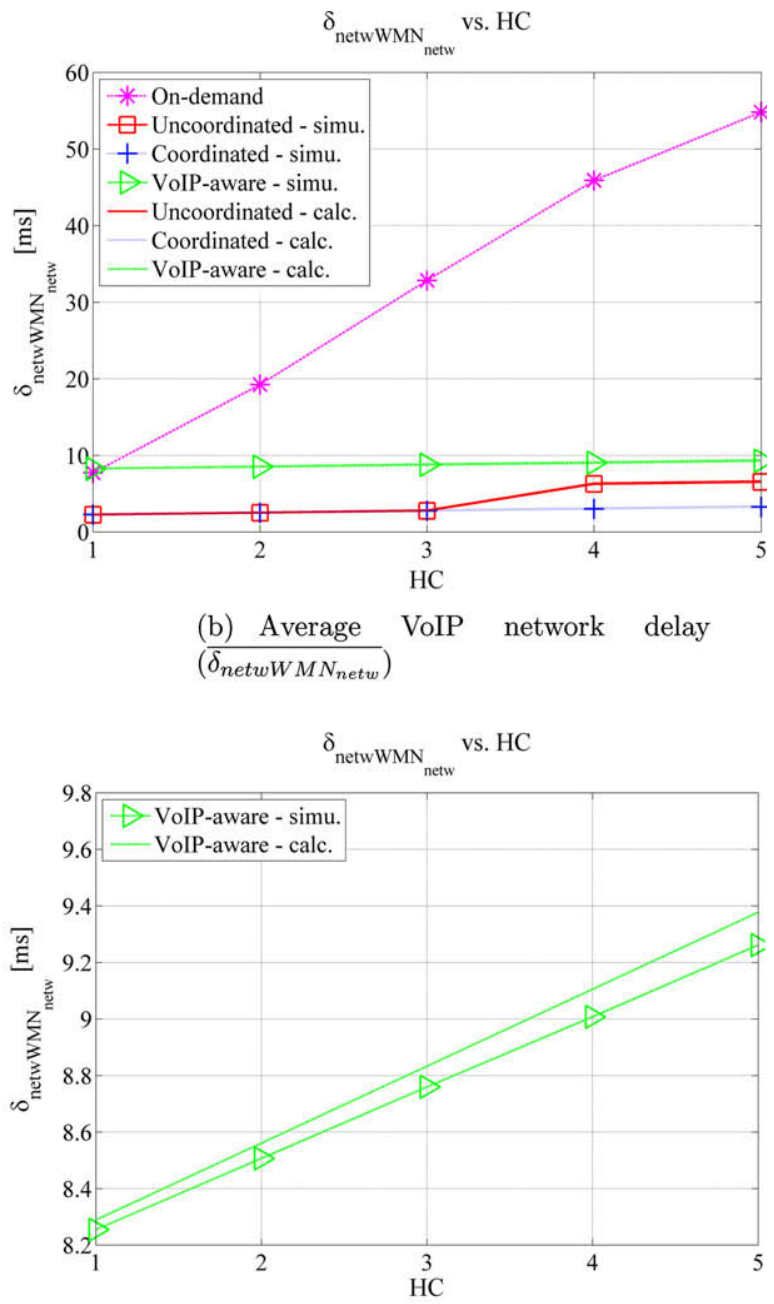

(d) Comparison of simulation and analytical results of the average network delay $\overline{\delta_{\text {netwWMN }} \text { netw }}$ for the VoIP-aware resource coordination scheme

Fig. 11. Quality of a single VolP session vs. the hop count $(H C)$ for the chain topology.

pendent of $\mathrm{HC}$ and very low in all cases. For both the unco ordinated and the coordinated scheme the standard devia tion is less than $1.2 \mathrm{~ms}$ and for the VoIP aware scheme it is less than $3 \mathrm{~ms}$.

In summary, it generally can be stated that for the chain topology all schedulers keep the delay of a single VoIP session very low. However, for the on demand scheduler, delay tends to increase linearly with $H C$ and as will be shown in Section 5.2, this leads to poor quality in more realistic scenarios. Using the continuous scheduler allows a network operator to provide minimum service guaran tees and to predict the expected network delay very accu rate as average delay as well as the delay standard deviation are very low. The coordinated and the VoIP aware resource coordination schemes are especially able to provide a high quality almost independent of the loca tion of the users (independent of $H C$ ).
5.1.1.2. Jitter results. Fig. 11c displays maximum jitter $\left(J_{\text {netw }}^{\max }\right)$ vs. hop count $(H C)$ and compares simulation results with the analytical results obtained from Eqs. (17) and (18).

Regarding the on demand scheduler, it can be seen that the maximum jitter linearly increases with $H C$ from $17.0 \mathrm{~ms}$ for $H C=1$ to $52.0 \mathrm{~ms}$ for $H C=5$. The reason for this is that the duration of the TWHS as well as the interval between TWHSs is not constant but varies significantly. As a TWHS must be performed for each packet and each link, delay varies from packet to packet. Therefore, jitter in creases over $H C$. Already for $H C$ 4, $J_{\text {netw }}^{\max }$ exceeded the threshold of $40 \mathrm{~ms}$ which means that some packets re ceived by the destination are outdated and will simply be dropped by the jitter buffer. In such situations a quality degradation can be expected.

Regarding the continuous scheduler, it is clear that sim ulation results match to the analytical results. In addition, 
the results show that, for all resource coordination schemes, $J_{\text {netw }}^{\max }$ is constant and independent of $H C$. The rea son for these promising results can be explained by the fact that a TDMA based MAC layer is used and that resources are continuously reserved. Thus, packets are sent and re ceived in constant time intervals.

Jitter results for the uncoordinated and the coordinated resource coordination scheme are equal. Maximum jitter has a constant and very small value of less than $4 \mathrm{~ms}$. This is due to the small time drift between the packet genera tion and the length of the TDMA frame. Some packets have arrived just after the transmission burst and have to be queued until the next frame.

$J_{\text {netw }}^{\max }$ for the VoIP aware resource coordination scheme is also constant but delayed by approximately $12 \mathrm{~ms}$. The reason for this is that packets that have arrived just after the transmission burst not only have to be queued for one frame but also for the transmission interval $I$.

The conclusions for the jitter results are the same as for the delay results. Using the on demand scheduler, maxi mum jitter increases linearly with $H C$ and, for connections with $H C \geqslant 4$, a degradation due to large jitter values is ex pected. In contrast, all resource coordination schemes of the continuous scheduler are able to provide very low maximum jitter in all cases, independent of HC. Hence, a quality degradation due to large jitter values is not expected.

5.1.1.3. Packet error rate results. It was found that VoIP impairment due to packet loss is not an issue in the chain topology. Hence, the results are not displayed but ex plained in this text.

For all resource coordination schemes of the continuous scheduling mechanism, $\overline{e_{n e t w}}$, is zero in all cases. First, the network load is very low and concurrent transmissions do not occur. Therefore, interference can be neglected and $e_{\text {trans }}$ is zero. Also $e_{j i t t e r}$ is zero as the maximum jitter never exceeds the critical threshold of $40 \mathrm{~ms}$.

Regarding the on demand scheduler this is slightly dif ferent. For $H C=1$ to $H C \quad 3, \overline{e_{\text {netw }}}$ is also zero, as parallel transmissions do not appear and because the maximum jitter is below $40 \mathrm{~ms}$. However, for larger $H C$ values this is different and $e_{\text {netw }}=0.06 \%$ for $H C=4$ and $e_{\text {netw }}=0.26 \%$ for $H C=5$. There are two reasons for this. The first is inter ference due to concurrent transmissions and the second is packet loss caused by large jitter values as explained in the previous section. However, $e_{\text {netw }}$ is still far below $1 \%$ which means that the VoIP quality will not be significantly reduced.

In summary, it can be stated, that for the chain topology with only one active VoIP session, a quality reduction due to packet errors is not an issue for all scheduling mechanisms.

5.1.1.4. $R$ score results. To summarise the previous find ings, Fig. 11a shows the average $R$ score $\left(\overline{R_{\text {netw }}}\right)$ vs. hop count $(H C)$ obtained through simulations as well as analyt ical investigations according to Eq. (3).

It can be seen that all schedulers are able to provide a very predictable quality with an average $R$ score $>90$. This means that users will be "very satisfied".
The on demand scheduler shows the worst perfor mance as $\overline{R_{\text {netw }}}$ decreases and $R$ score standard deviation (not shown) increases by increasing $H C$. For $H C=1$ the $R$ score value is 92.6 and for $H C=5$ it is 90.3 . These results are as expected and are due to the increased delay, jitter and transmission errors as discussed in the previous sec tions. As a result, the on demand scheduler causes unfair ness as users that are close to the mesh base station will have a better VoIP quality compared to users further from the mesh base station. Furthermore, it is expected, that the performance of the on demand scheduler and hence the VoIP quality will be worse in larger networks and more realistic scenarios.

The continuous scheduler provides very high quality for all resource coordination schemes and the $R$ score value is always greater than 92. In addition, the coordinated scheme as well as the VoIP aware scheme are able to fulfil carrier grade requirements as they provide equal quality, independent of HC. Hence, a high VoIP quality can be pro vided independent of the location of the user. Also the $R$ score standard deviation (not shown) is very small which makes the system highly predictable and gives the net work operator the possibility to determine the VoIP quality already in the planning phase of the network.

\subsubsection{Scalability investigations}

In this section the amount of VoIP traffic that can be supported in the chain topology is investigated. The sink for the VoIP streams is always set to node 0 while the source is varied in order to simulate different hop counts. The simulation time is set to $45 \mathrm{~s}$. The first VoIP session is started at $t=(5+R) \mathrm{s}$. The interval between subsequent sessions is $(0.5+R N D) \mathrm{s}$. $R N D$ is a uniformly distributed random value between 0 and 1 .

In these experiments, the network is loaded with the maximum possible number of VoIP sessions. The maxi mum number of sessions is limited by the Call Admission Control (CAC) mechanism.

CAC is a very useful tool for network operators to en sure that networks are not overloaded in order to maintain the high quality for each session. CAC is not the focus of this paper and therefore, only a simple mechanism has been implemented. This mechanism consists of two poli cies that block new VoIP calls:

- Resource policy: No MSs are available for transmission on one of the links from the source to the destination.

- Quality policy: The $R$ score of one of the running VoIP sessions drops below a defined threshold $\left(R_{T h r}\right)$.

The scalability results are shown in Table 3. Besides the maximum number of calls that can be supported $(C)$, the delay, delay impairment, jitter, jitter impairment, loss, loss impairment and $R$ score are listed and compared for differ ent hop count values. For the on demand scheduler it can be seen that a large number of calls can be supported if $H C$ is small. However, by increasing $H C$, scalability de creases exponentially and less VoIP calls are supported compared to all other schedulers. Also the average $R$ score is close to the threshold of 90 . It was found that, due to the first come first serve packet treatment applied by the 
Table 3

Scalability results for the chain scenario.

\begin{tabular}{|c|c|c|c|c|c|c|c|c|c|}
\hline Scheduler & HC & $\overline{\delta_{\text {netw }}}(\mathrm{ms})$ & $\overline{I_{d}}$ & $j(\mathrm{~ms})$ & $\overline{e_{j i t t e r}}$ & $\overline{e_{\text {netw }}}$ & $\overline{I_{e f}}$ & $\bar{R}$ & $C$ \\
\hline \multirow[t]{5}{*}{ On-demand } & 1 & 75.46 & 3.60 & 2.85 & 0 & 0 & 0 & 90.6 & 58 \\
\hline & 2 & 72.15 & 3.52 & 4.66 & 0 & 0 & 0 & 90.7 & 29 \\
\hline & 3 & 62.20 & 3.28 & 8.74 & $0.40 E-3$ & 0 & 0.19 & 90.7 & 17 \\
\hline & 4 & 48.78 & 2.96 & 9.65 & $1.00 \mathrm{E}-3$ & $0.50 \mathrm{E}-3$ & 0.67 & 90.6 & 4 \\
\hline & 5 & 54.89 & 3.11 & 10.51 & 0 & $1.10 \mathrm{E}-3$ & 0.48 & 90.6 & 2 \\
\hline \multirow[t]{5}{*}{ Un-coordinated } & 1 & 2.23 & 1.84 & 0.08 & 0 & $2.59 \mathrm{E}-4$ & 0.12 & 92.2 & 12 \\
\hline & 2 & 2.46 & 1.85 & 0.08 & 0 & $5.93 \mathrm{E}-4$ & 0.27 & 92.1 & 6 \\
\hline & 3 & 2.69 & 1.85 & 0.08 & 0 & $8.00 \mathrm{E}-4$ & 0.36 & 91.9 & 4 \\
\hline & 4 & 6.24 & 1.94 & 0.08 & 0 & $1.14 \mathrm{E}-3$ & 0.51 & 91.8 & 4 \\
\hline & 5 & 6.47 & 1.95 & 0.08 & 0 & $1.47 \mathrm{E}-3$ & 0.65 & 91.6 & 4 \\
\hline \multirow[t]{5}{*}{ Coordinated } & 1 & 2.23 & 1.84 & 0.08 & 0 & $2.49 \mathrm{E}-4$ & 0.14 & 92.3 & 12 \\
\hline & 2 & 2.46 & 1.85 & 0.08 & 0 & $5.25 \mathrm{E}-4$ & 0.29 & 92.1 & 6 \\
\hline & 3 & 2.68 & 1.85 & 0.08 & 0 & $8.45 \mathrm{E}-4$ & 0.38 & 92.0 & 4 \\
\hline & 4 & 3.23 & 1.87 & 0.08 & 0 & $2.70 \mathrm{E}-3$ & 1.19 & 91.1 & 4 \\
\hline & 5 & 3.46 & 1.87 & 0.08 & 0 & $4.07 E-3$ & 1.77 & 90.6 & 4 \\
\hline \multirow[t]{5}{*}{ VoIP-aware } & 1 & 8.24 & 1.99 & 5.96 & 0 & $3.23 E-4$ & 0.14 & 92.1 & 48 \\
\hline & 2 & 8.47 & 1.99 & 5.96 & 0 & $6.56 \mathrm{E}-4$ & 0.29 & 91.9 & 24 \\
\hline & 3 & 8.70 & 2.00 & 5.96 & 0 & $9.88 \mathrm{E}-4$ & 0.44 & 91.8 & 16 \\
\hline & 4 & 12.25 & 2.08 & 5.97 & 0 & $2.29 \mathrm{E}-3$ & 1.30 & 90.8 & 16 \\
\hline & 5 & 12.48 & 2.09 & 5.97 & 0 & $4.72 \mathrm{E}-3$ & 2.04 & 90.1 & 16 \\
\hline
\end{tabular}

on demand scheduler, the number of calls is limited by the CAC quality policy. This can also be seen by looking at the delay. Delay values for the 1 and 2 hop scenario are kept on a level close to the upper limit. In the other scenarios loss impairment exists which in turn requires a lower delay in order to keep the $R$ score above $R_{T h r}$. Thus, the number of supported calls is reduced which in turn decreases delay due to decreased network contention.

Regarding the delay, jitter and VoIP quality for the con tinuous resource coordination schemes, the results are more or less equal to the results discussed in the previous section with only one active VoIP session. The only differ ence which can be observed is the increased packet loss that slightly decreases VoIP quality. This loss is caused by the increased interference due to the highly loaded net work. Therefore, it is clear and has also been observed, that the number of supported calls for the continuous resource coordination scheme is limited by the CAC resource policy. The VoIP aware resource coordination scheme shows good performance in all scenarios. Especially if $H C$ is large, it outperforms all other schedulers and is able to improve the number of supported calls by at least a factor of four.

\subsection{Grid network topology}

In this section the performance of the different schedul ers in the grid scenario is discussed. In contrast to the chain topology the grid topology provides an insight into the behaviour in more realistic scenarios.

\subsubsection{Influence of the number of parallel VoIP sessions}

In this section the amount of VoIP traffic which can be supported and the influence on the VoIP quality is investi gated. For this investigation, the grid topology as shown in Fig. 10 was chosen, and for the VoIP connections, the sink is set to node 12 while the sources are uniformly distributed across all nodes in the mesh network. The first stream is started at $t=(7+R) \mathrm{s}$ and the interval between subsequent sessions is $(0.5+R N D) \mathrm{s}$ where $R N D$ is a uniformly distrib uted random value between 0 and 1 . The duration of each simulation is $35 \mathrm{~s}$. In addition to the VoIP traffic, TCP traffic is also sent between node 12 and the edge nodes node 0 and node 24 . This TCP traffic is, in all cases, served by the on demand scheduler. The starting time for each TCP ses sion is $t=(5+R N D) \mathrm{s}$

Fig. 12b displays the average number of supported VoIP sessions $\left(\overline{C_{\text {supported }}}\right)$ vs. the number of offered VoIP sessions $\left(C_{\text {offered }}\right)$. The shape of the curve is equal for all continuous schedulers. If the network is loaded only with a few VoIP sessions, all can be supported and the curves increase line arly. However, at a certain point, the curves begin to satu rate. This can be explained by the explicit resource reservation of the continuous scheduling mechanism. In case all resources are reserved the CAC resource policy re jects new calls. It can be seen that for the VoIP aware re source coordination scheme, the saturation point comes much later compared to the other schemes. While the uncoordinated and coordinated scheme, in average, sup port a maximum of 5.7 calls, the VoIP aware scheme shows a significant improvement and supports an average of 22.1 calls. This can be explained by the application awareness and thus efficient resource assignment of the VoIP aware resource coordination scheme. It should be noted that this increased scalability has no negative influence on the VoIP quality. The average $R$ score values $\left(\overline{R_{\text {netw }}}\right)$ as displayed in Fig. $12 \mathrm{a}$ are very close for all continuous resource coordina tion schemes and almost independent of $C_{\text {offered }}$ due to the continuous and explicit MS reservation. As the VoIP quality provided by the continuous scheduler in the grid topology is equal to the quality provided in the chain topology, a con clusion can be made that the TCP background traffic does not have a negative influence on the VoIP traffic. A further importance is that the $R$ score standard deviation (not dis played) is very low, making the system very predictable.

These very good quality results are also reflected in Fig. 12e, $\mathrm{c}$ and $\mathrm{d}$ in which the average delay $\left(\overline{\delta_{\text {netwWMN }}{ }_{\text {netw }}}\right)$, 


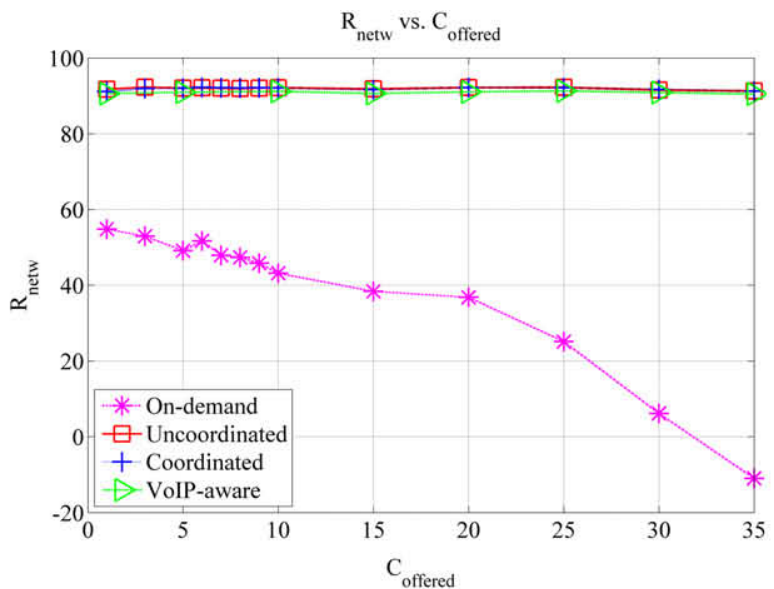

(a) Average VoIP R-score $\left(\overline{R_{\text {netw }}}\right)$

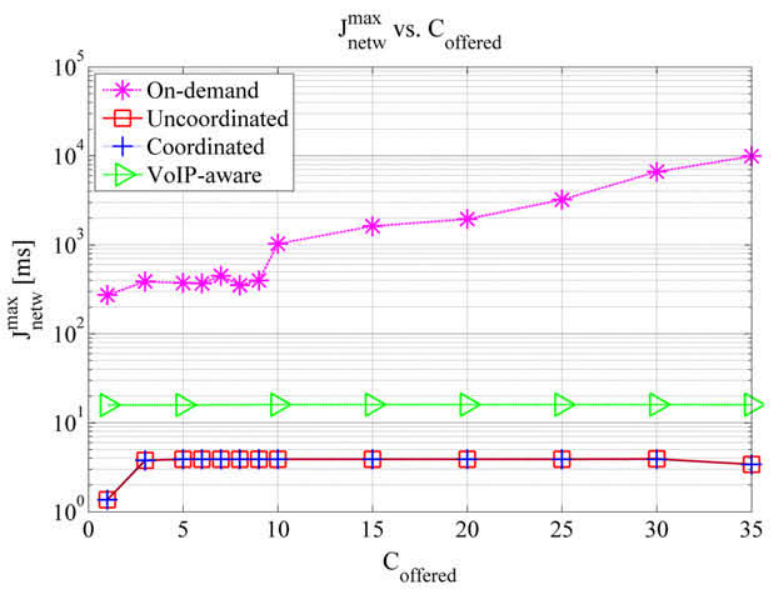

(c) Max. VoIP packet jitter $\left(J_{\text {netw }}^{\max }\right)$

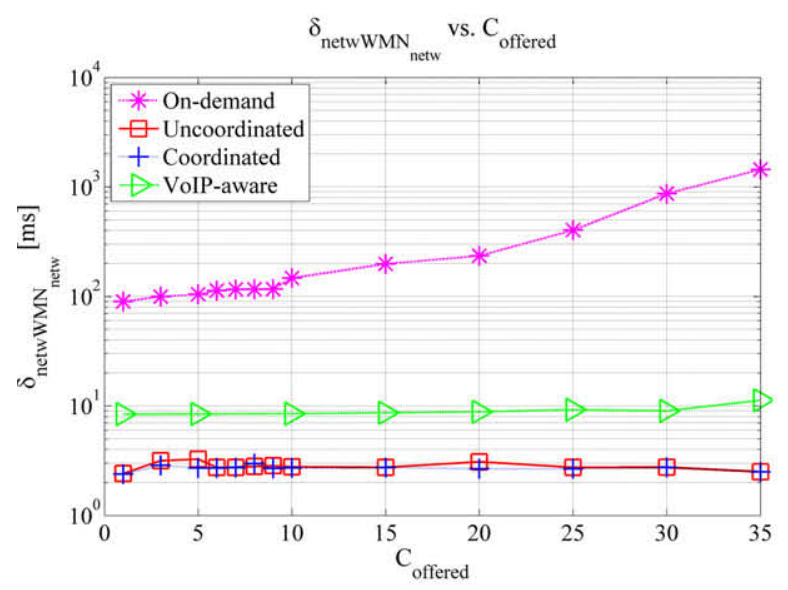

(e) Average VoIP network delay $\left(\overline{\delta_{\text {netwWMN }} N_{\text {netw }}}\right)$

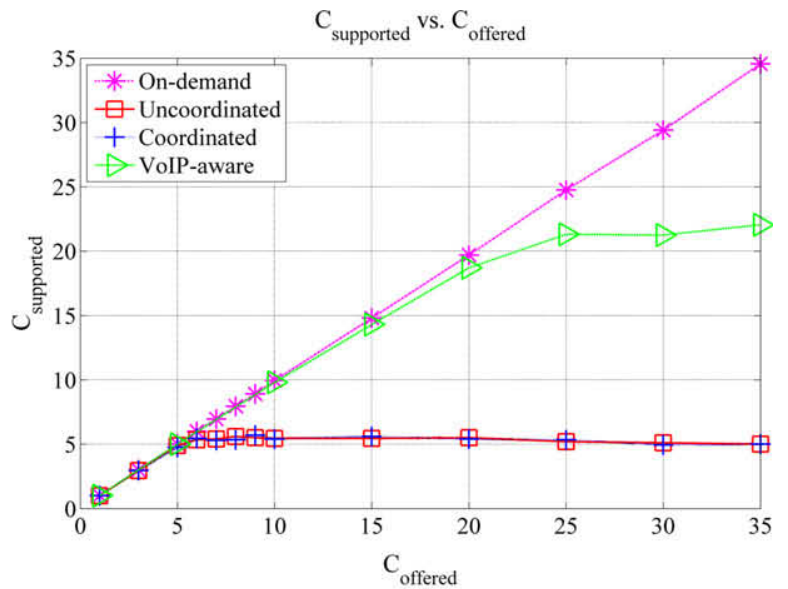

(b) Average number of supported VoIP sessions $\left(\overline{C_{\text {supported }}}\right)$

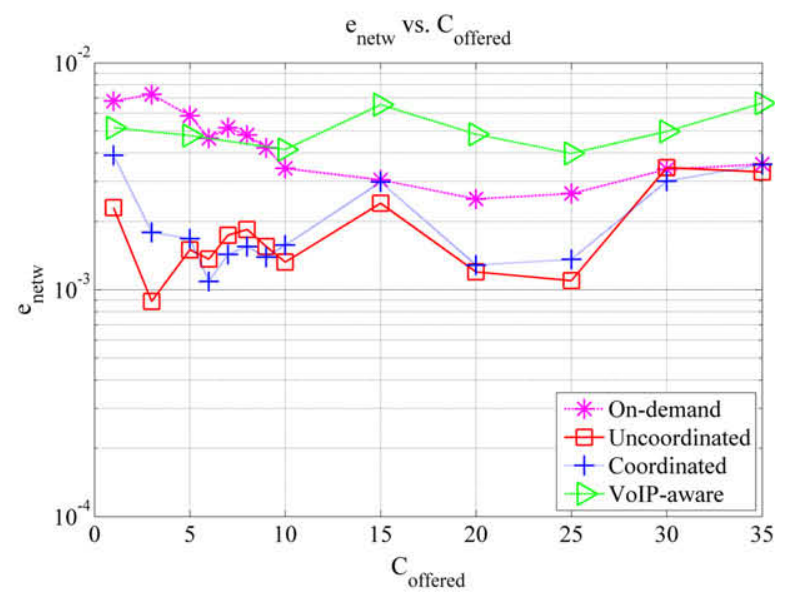

(d) Average VoIP PER $\left(\overline{e_{\text {netw }}}\right)$

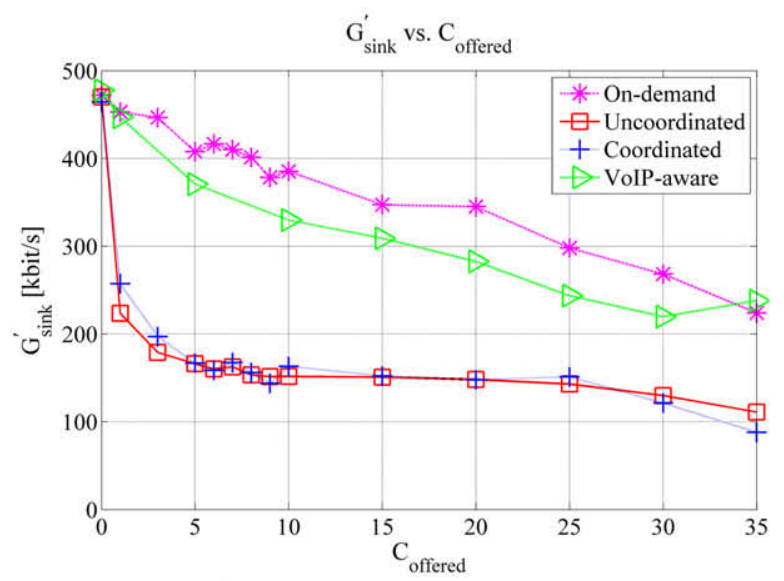

(f) Average aggregated TCP throughput $\left(\overline{G_{\text {sink }}^{\prime}}\right)$

Fig. 12. Average VoIP quality vs. the number of offered VoIP sessions $\left(C_{\text {offered }}\right)$ for the grid topology.

maximum jitter $\left(J_{\text {netw }}^{\max }\right)$ and average PER $\left(\overline{e_{\text {netw }}}\right)$ are dis played. The delay results reflect the results for the chain scenarios. $\overline{\delta_{\text {netwWMN }} \text { netw }}$ is constant and independent of the number of offered VoIP sessions. Again, the delay of 
the VoIP aware resource coordination scheme is slightly larger compared to the other resource coordination schemes. However, this is the cost for the significantly im proved scalability as previously discussed. Also $J_{\text {netw }}^{\max }$ does not depend on the number of offered VoIP sessions. For all schedulers, jitter is more or less constant. The VoIP aware scheme, again, has a slightly increased jitter com pared to the uncoordinated and the coordinated schemes. However, jitter is for all continuous resource coordination schemes very low and a quality degradation is not to be ex pected. The PER results are also as expected. In general, $\overline{e_{\text {netw }}}$ slightly increases with increasing $C_{\text {offered }}$ as this, in turn, increases the amount of traffic in the network and thus, the interference caused by simultaneous transmis sions. $\overline{e_{\text {netw }}}$ is equal for the uncoordinated and the coordi nated scheme and smaller compared to the VoIP aware scheme. The explanation for this is simple. As the VoIP aware scheme increases the number of supported VoIP streams by a factor of four compared to the other schemes, the load in the network is higher. Therefore, interference due to simultaneous transmissions increases.

For the on demand scheduler, the CAC quality policy has been switched off for the investigations discussed here. It is then clear that due to the first come first serve treat ment of packets, the number of supported calls does not saturate. However, regarding the average $R$ score, this scheduler causes bad VoIP quality in all scenarios far below an acceptable level. This is mainly due to large delay and large jitter values as shown in Fig. 12e and c. Furthermore, $\overline{\delta_{\text {netwWMN }} \text { netw }}$ is constantly increasing with $C_{\text {offered }}$. This is nat ural as the network has only a limited capacity. Therefore, if the amount of traffic increases, the time a packet has to wait in the queue until it can be transmitted increases. Although, even for a single VoIP session the quality is bad. There are two reasons for this. Firstly, in comparison with the chain topology the grid topology is denser and more nodes compete for channel access which increases the average TWHS duration. Secondly, the TCP background traffic increases the contention as well. All these facts lead to a bad and unpredictable VoIP quality.

In Fig. $12 \mathrm{f}$ the aggregated TCP traffic at the mesh base station vs. the number of offered VoIP sessions is shown in order to highlight the influence that the different sched ulers have on the data traffic which is served by the on de mand scheduler. It can be seen that, for all schedulers, TCP throughput linearly decreases up to a point after which, it saturates and remains constant. These results are as ex pected due to the link sharing model which is imple mented to share the MSs amongst the different schedulers in times of congestion. The link sharing model comprises different policies. In our example a fixed amount of resources is allocated to each scheduler. $z$ is the amount of resources for the on demand scheduler and $(100 \quad z)$ is the amount of resources for the continuous scheduler which can be obtained by each scheduler in times of congestion. Thereby, network operators are able to provide a minimum service guarantee even in heavily loaded networks. In times in which the network is not con gested and in which one of the schedulers does not fully use the allocated resources, the following policies define how to handle these "excess" resources:
- To increase the efficiency of the network, excess resources of the continuous scheduler can be used by the on demand scheduler.

- To increase the quality and scalability of VoIP traffic, excess resources of the on demand scheduler cannot be used by the continuous scheduler. Otherwise VoIP sessions would be terminated when a new FTP session is added.

To ensure, that the excess resources of the continuous scheduler, used by the on demand scheduler, do not block new calls, the frame lookup (FrLo) parameter has been de fined. FrLo is used in the following ways:

- The on demand scheduler is only allowed to request/ assign resources within frames $(x)$ for which: CurrentFrameNumber $\leqslant x<$ FrameCount + FrLo.

- The continuous scheduler always searches for resources in frames $(x)$ for which: $x \geqslant$ FrameCount + FrLo.

Thus, it is clear that TCP claims all of the resources in case no VoIP sessions are active. As resources used by VoIP cannot be used by TCP, the TCP throughput linearly de creases with increasing number of VoIP sessions. For all schedulers TCP throughput will at some point saturate and this saturation comes much earlier for the uncoordi nated and coordinated schemes as for the VoIP aware scheme. This is in line with the previous results. As only a specific number of VoIP sessions can be supported by each resource assignment scheme, further increasing the number of offered sessions has no additional influence on TCP throughput as these sessions will be rejected due to the lack of available resources. It should be noted that, in general, the VoIP aware scheme allows higher TCP rates compared to the other schemes. This is because of the effi cient resource usage of the VoIP aware scheme by using application information.

\subsubsection{Influence of the amount of background TCP traffic}

In this section the influence of the background traffic on VoIP performance is investigated. The amount of back ground traffic is varied by changing the number of offered TCP sessions $\left(\right.$ TCP $\left._{\text {offered }}\right)$. The TCP sink is set to node 12 (see Fig. 10) while the sources are uniformly distributed across all nodes in the mesh network. The first TCP session is started at $t=(7+R N D) \mathrm{s}$ and the interval between subse quent sessions is $(0.5+R N D)$ s. In parallel to the TCP traffic two VoIP sessions are simulated. The sink of these sessions is set to node 12 while the source nodes are node 0 and node 24. The first VoIP session is started at $t=(5+R N D) \mathrm{s}$ and the second session is started at $t=(5.5+R N D) \mathrm{s}$. $R N D$ is a uniformly distributed random value between 0 and 1. The simulation time is $20 \mathrm{~s}$. The TCP traffic is served by the on demand scheduler and no CAC mechanism is ap plied for the TCP traffic.

Fig. 13b shows the average aggregated TCP throughput measured at the sink $\left(\overline{G_{\text {sink }}^{\prime}}\right)$ vs. the number of offered TCP sessions $\left(T C P_{\text {offered }}\right)$. In principle the results of the uncoordi nated and the coordinated schemes are identical. This is clear as both schemes do not take into consideration how efficiently resources are assigned but only how resources 


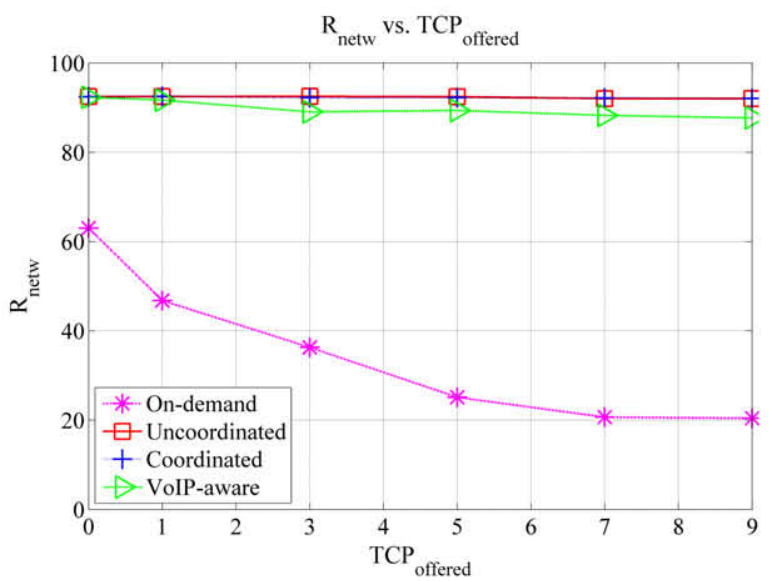

(a) Average VoIP R-score $\left(\overline{R_{n e t w}}\right)$

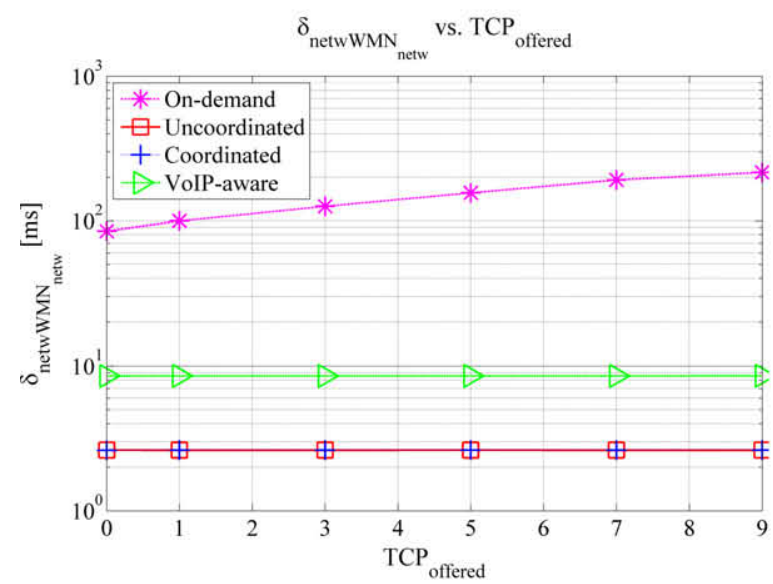

(c) Average VoIP network delay $\left(\overline{\delta_{\text {netw } W M N_{\text {netw }}}}\right)$

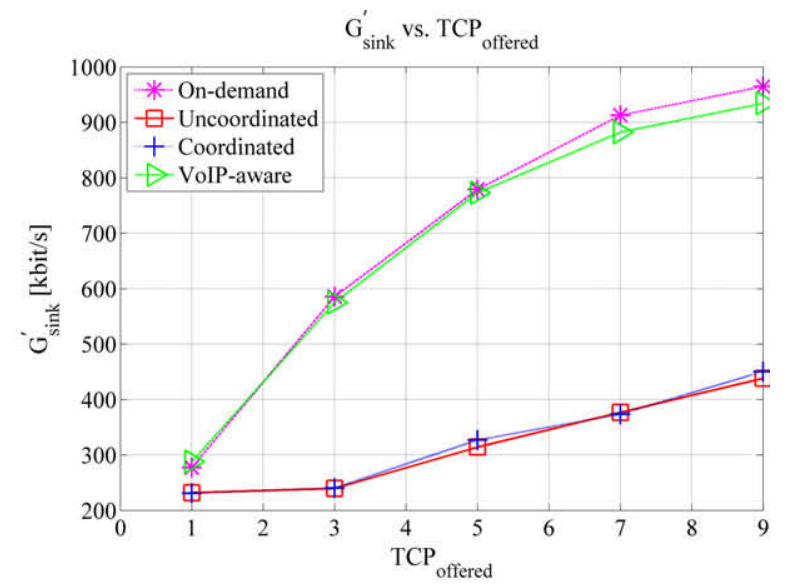

(b) Average aggregated TCP throughput $\left(\overline{G_{\text {sink }}^{\prime}}\right)$

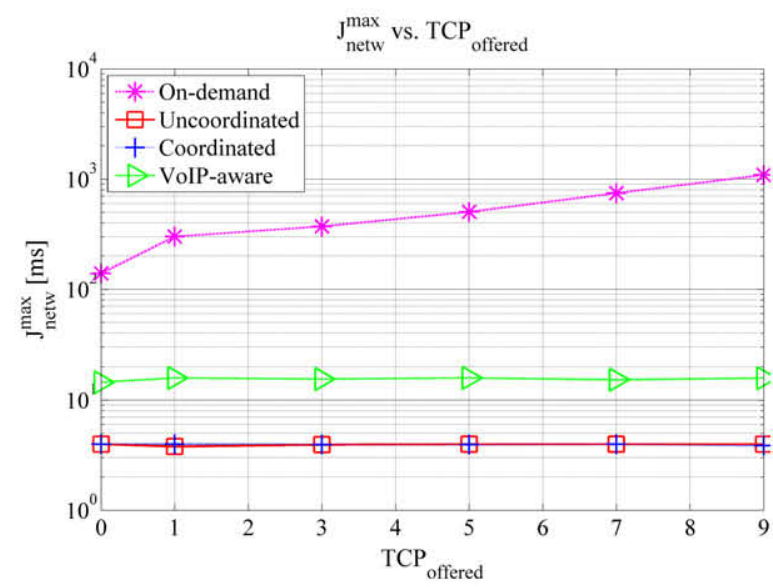

(d) Max. VoIP packet jitter $\left(J_{n e t w}^{\max }\right)$

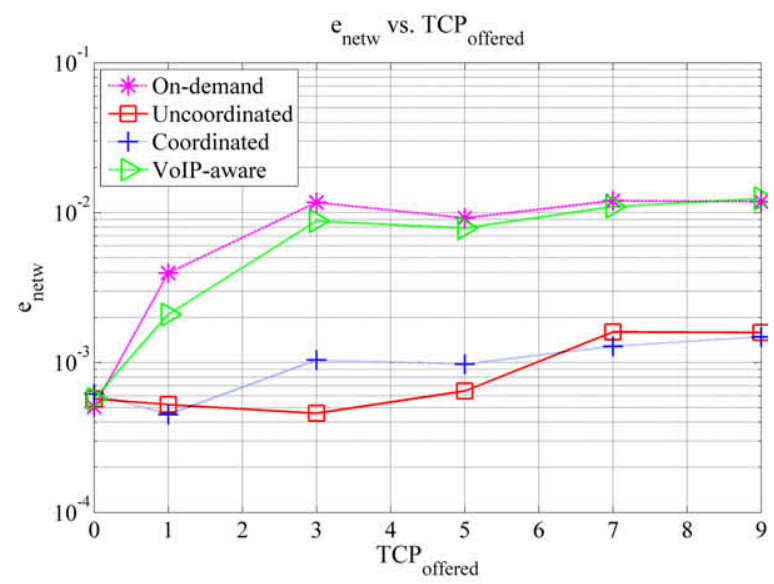

(e) Average VoIP PER $\left(\overline{e_{n e t w}}\right)$

Fig. 13. Average VoIP quality vs. the number of offered TCP connections ( $\left.T C P_{\text {offered }}\right)$ for the grid topology.

are coordinated. Hence, the amount of resources that can be used for the transmission of TCP packets is equal. Also the results of the VoIP aware scheme and the on demand scheduler are identical but the performance is much higher compared to the two other schemes. For the VoIP aware scheme this is because of the efficient resource reservation 
which considers the VoIP packet interval. For the on de mand scheduler, resources are not reserved explicitly and packets are treated on a first come first serve basis. The dis advantage is very poor VoIP quality as shown in Fig. 13a. In none of the cases is the on demand scheduler able to pro vide an acceptable quality. This is different for the contin uous scheduling mechanism and a very high quality can be guaranteed nearly independent of the amount of TCP traf fic in the network. Only for the VoIP aware resource coor dination scheme the $R$ score slightly decreases as the amount of TCP traffic increases. Once again the $R$ score standard deviation (not shown) is very low for all the re source coordination schemes of the continuous scheduler.

Average delay, maximum jitter and average PER are dis played in Fig. $13 \mathrm{c}$ e. The same conclusion can be drawn for all the resource coordination schemes of the continuous scheduling mechanism as in the previous sections. $\overline{\delta_{\text {netw } W M N_{\text {netw }}}}$ and $J_{\text {netw }}^{\max }$ are constant and not influenced by the amount of background traffic due to the explicit re source reservation. Again, the cost of the increased scala bility of the VolP aware scheme is slightly increased delay and jitter values. However, this does not cause any significant quality reduction. Looking at $\overline{e_{n e t w}}$ it is found that PER slightly increases by increasing the amount of background traffic due to the fact that this increases the interference. For the on demand scheduler and the VoIP aware scheme this increase is larger compared to the other schemes. The reason is that the on demand scheduler as well as the VoIP aware scheme have a much better scala bility and thus the load in the network is much higher, increasing the overall interference. This increased PER is the reason why the $R$ score of the VoIP aware scheduler slightly decreases by increasing the amount of TCP traffic. The bad VoIP quality using the on demand scheduler is mainly caused by the increased average delay and the in creased maximum jitter.

\section{Conclusions}

This paper analyses the quality and scalability of VoIP in TDMA based WMNs. Different scheduling approaches are investigated and compared, by measuring the most impor tant metrics for voice: delay, jitter, packet loss as well as the number of supported calls. The main findings of the pa per are: (1) On demand scheduling of VoIP traffic as used in Wireless Local Area Network (WLAN) based WMNs makes no sense from a network operator point of view as it is not able to provide good VoIP quality in a realistic network sce nario. (2) Continuous scheduling is well suited to serve VoIP traffic. (3) The optimised VoIP aware scheduling scheme provides carrier grade services and high VoIP quality, inde pendent of the number of hops between the node and the mesh base station, the number of parallel VoIP sessions and the amount of background traffic within the network (4) Application aware scheduling, as used by the VoIP aware resource coordination scheme, is able to significantly increase scalability due to the efficient resource assignment.

This paper shows that, contrary to popular belief, mesh networks are able to support VoIP with good quality when a continuous scheduler is applied. From the view of a net work operator the VoIP aware resource coordination scheme seams to be the best choice as it provides good voice quality far below the upper bounds of delay, jitter and PER and is also very efficient and able to support a large number of simultaneous connections.

Objectives for future work are to implement the VoIP aware resource coordination scheme in a mesh test bed in order to investigate its performance in a realistic environment.

\section{Acknowledgement}

The research leading to these results has received fund ing from the European Community's Seventh Framework Programme (FP7/2007 2013) under grant agreement no. 214994.

\section{References}

[1] Belair Networks Webpage. <http://www.belairnetworks.com/>.

[2] Saxnet Webpage. <http://www.meshnode.org/>.

[3] Cisco Systems Webpage. <http://www.cisco.com/>.

[4] Roofnet Webpage. <http://pdos.Ics.mit.edu/roofnet/>.

[5] Freifunk Webpage. <http://www.freifunk.net/>.

[6] R. Karrer, P. Zerfos, N. Piratla, Magnets - a next generation access network, in: Poster at IEEE INFOCOM 2006, April 2006.

[7] R. Dilmaghani, B. Manoj, B. Jafarian, R. Rao, Performance evaluation of RescueMesh: a metro-scale hybrid wireless network, in: WiMesh 2005: First IEEE Workshop on Wireless Mesh Networks, September 2005.

[8] N. Bayer, D. Hock, A. Roos, M. Siebert, B. Xu, V. Rakocevic, J. Habermann, VoIP performance in "MeshBed" - a wireless mesh networks testbed, in: Proceedings of the IEEE 67th Vehicular Technology Conference (VTC'08-Spring), Marina Bay, Singapore, May 2008.

[9] The Institute of Electrical and Electronics Engineers, IEEE Standard for Local and Metropolitan Area Networks Part 16: Air Interface for Fixed Broadband Wireless Access Systems, IEEE Std 802.16-2004, IEEE, 2004.

[10] International Organization for Standardization, Information Technology - Telecommunications and Information Exchange Between Systems - Local and Metropoloitan Area Networks Specific Requirements - Part 11: Wireless LAN Medium Access Control (MAC) and Physical Layer (PHY) Specifications.

[11] D. Hock, N. Bayer, R. Pries, M. Siebert, D. Staehle, V. Rakocevic, B. Xu, QoS provisioning in WLAN mesh networks using dynamic bandwidth control, in: European Wireless 2008, Prague, Czech Republic, June 2008, p. 7.

[12] D. Zhao, X. Shen, Performance of packet voice transmission using IEEE 802.16 protocol, Wireless Commun. Mag., IEEE (2007) 44-51.

[13] S. Sengupta, S. Sengupta, M. Chatterjee, S. Ganguly, Improving quality of VoIP streams over WiMax, IEEE Trans. Comput. 57 (2) (2008) 145-156.

[14] B.H. Lee, G.Y. Cai, Y. Ge, W.K. Seah, VoIP capacity over wireless mesh networks, in: Proceedings 2006 31st IEEE Conference on Local Computer Networks, Tampa, FL, USA, November 2006, pp. 151-152.

[15] D. Niculescu, S. Ganguly, K. Kim, R. Izmailov, Performance of VoIP in an 802.11-based wireless mesh network, in: IEEE INFOCOM, Barcelona 2006, 2006.

[16] K. Kim, S. Ganguly, R. Izmailov, S. Hong, On Packet aggregation mechanisms for improving VoIP quality in mesh networks, in: Vehicular Technology Conference, 2006, VTC 2006-Spring, IEEE 63rd, vol. 2, 2006, pp. 891-895.

[17] J. Lu, K. Tan, Q. Zhang, Path aggregation for voice over IP in multihop wireless mesh networks, This paper appears in: Communications, 2006, ICC'06, IEEE International Conference, vol. 9, 2006, pp. 3990 3995.

[18] M. Cao, V. Raghunathan, P.R. Kumar, A tractable algorithm for fair and efficient uplink scheduling of multi-hop WiMax mesh networks, in: Proceedings of the WiMesh 2006: Second IEEE Workshop on Wireless Mesh Networks, Washington, DC, USA, September 2006.

[19] J. Chen, C. Chi, Q. Guo, A bandwidth allocation model with high concurrence rate in IEEE802.16 mesh mode, in: Proceedings of the Asia-Pacific Conference, October 2005, pp. 750-754 
[20] L. Fu, Z. Cao, P. Fan, Spatial reuse in IEEE 802.16 based wireless mesh networks, in: IEEE Communications and Information Technology, 2005, ISCIT 2005, vol. 2, October 2005, pp. 1358-1361.

[21] J. Tao, F. Liu, Z. Zeng, Z. Lin, Throughput enhancement in WiMax mesh networks using concurrent transmission, in: Wireless Communications, Networking and Mobile Computing, 2005, Proceedings, vol. 2, September 2005, pp. 871-874.

[22] H.-Y. Wei, S. Ganguly, R. Izmailov, Z.J. Haas, Interference-aware IEEE 802.16 WiMax mesh networks, in: Vehicular Technology Conference, 2005, VTC 2005-Spring, 2005 IEEE 61st, vol. 5, 2005, pp. 3102-3106.

[23] C. Schwingenschlögl, P. Mogre, M. Hollick, V. Dastis, R. Steinmetz, Performance analysis of the real-time capabilities of coordinated centralized scheduling in 802.16 mesh mode, in: Proceedings of 63th IEEE Semiannual Vehicular Technology Conference (VTC-Spring 2006), Melbourne, Australia, May 2006.

[24] M. Lott, S. Redana, M. Carlozzo, Reducing the delay of IEEE 802.16a in multi-hop scenarios, in: Proceedings of the Sixth World Wireless Congress WWC 2005, San Francisco, CA, USA, May 2005.

[25] I.F. Akyildiz, X. Wang, W. Wang, Wireless mesh networks: a survey, Comput. Network 47 (4) (2005) 445-487. <http://portal.acm.org/ citation.cfm?id=1071646> (Online).

[26] S.-Y. Wang, C.-C. Lin, H.-W. Chu, T.-W. Hsu, K.-H. Fang, Improving the performances of distributed coordinated scheduling in IEEE 802.16 mesh networks, IEEE Trans. Veh. Technol. 57 (4) (2008) 2531-2547.

[27] M. Cao, W. Ma, Q. Zhang, X. Wang, W. Zhu, Modelling and performance analysis of the distributed scheduler in IEEE 802.16 mesh mode, in: MobiHoc'05: Proceedings of the Sixth ACM International Symposium on Mobile Ad hoc Networking and Computing, New York, NY, USA, ACM Press, 2005, pp. 78-89.

[28] 3GPP2 (2005): 3rd Generation Partnership Project, TS 23.228: Release 7, IP Multimedia Subsystem (IMS).

[29] ETSI ES 282 003: Telecommunications and Internet Converged Services and Protocols for Advanced Networking (TISPAN); Resource and Admission Control Sub-system (RACS); Functional Architecture.

[30] ITU-T Recommendation G.107, The E-model, A Computational Model for Use in Transmission Planning, International Telecommunication Union, Tech. Rep., 2003.

[31] R.G. Cole, J.H. Rosenbluth, Voice over IP performance monitoring, SIGCOMM Comput. Commun. Rev. 31 (2) (2001) 9-24.

[32] S. McCanne, S. Floyd, ns Network Simulator. <http://www.isi.edu/ nsnam/ns/>.

[33] J. Khun-Jush, P. Schramm, U. Wachsmann, F. Wenger, Structure and performance of the HIPERLAN/2 physical layer, in: Vehicular Technology Conference, 1999, VTC 1999 - Fall, IEEE VTS 50th, September 1999, pp. 2667-2671.

[34] V. Erceg, K. Hari, M. Smith, D. Baum, et al., Channel models for fixed wireless applications, IEEE 802.16.3 Task Group Contributions, Doc. IEEE 802.16.3.c-01/29r4, 2001.

[35] V. Erceg, L. Greenstein, S. Tjandra, S. Parkoff, A. Gupta, B. Kulic, A. Julius, R. Jastrzab, An empirically-based path loss model for wireless channels in suburban environments, in: Global Telecommunications Conference, 1998, GLOBECOM 98, The Bridge to Global Integration, IEEE, vol. 2, 1998

[36] J. Robinson, E.W. Knightly, A performance study of deployment factors in wireless mesh networks, in: INFOCOM, 2007, pp. 20542062.

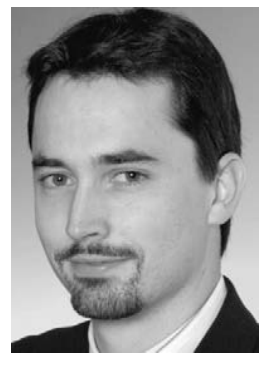

Nico Bayer received his diploma degree in Electrical Engineering and Information Technology from the University of Applied Sciences Friedberg, Germany, in 2003 and his $\mathrm{Ph} . \mathrm{D}$. in Electrical Engineering from the City University London, UK, in 2009. From 2003 till 2008 he worked for T-Systems Enterprise Services $\mathrm{GmbH}$ in the department of "Mobile and Wireless Solutions". Since 2008 he is working for Deutsche Telekom Laboratories in the department of "Seamless Communication" in Darmstadt, Germany. He is involved in different national and international R\&D projects in the fields of mobile communications, mesh networks and Fixed/Mobile Convergence (FMC).

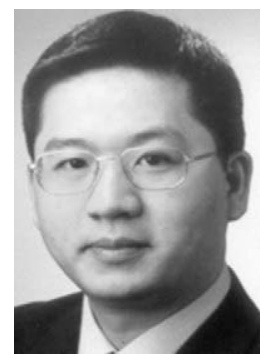

Bangnan $\mathbf{X u}$ is a Research Expert at T-Systems, Deutsche Telekom, leading various research projects in ad hoc/mesh networks, seamless IP mobility, fixed \& mobile convergence, and next generation access networks. His current research interests include Next Generation Mobile Network (NGMN, 3GPP LTE SAE), Next Generation Network (NGN, ETSI TISPAN), IMS and beyond, packet optimised fixed \& mobile converged (FMC), and Quadrupple play services. He received his Ph.D. in Electrical Engineering and Information Technology from Aachen Univ. of Technology (2002), as well as M.Sc. (1989) and B.Sc. (1986) degrees in Electrical Engineering from Dalian Maritime University. From 1989 to 1996, he was a lecturer on communication systems in Dalian Maritime University. Before joining T-Systems in 2001, he was with the chair of Communication Networks, Prof. Dr.-Ing. B. Walke, Aachen Univ. of Technology, Aachen, Germany. He holds several patents in multihop communications, ad hoc routing, and seamless IP mobility. He has authored and coauthored many technical papers, three of them granted with Best Paper Award.

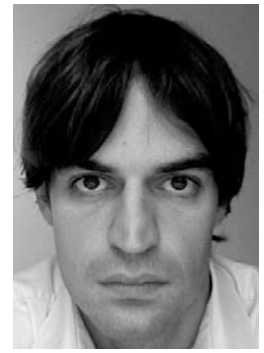

Veselin Rakocevic MIEEE currently works as a Senior Lecturer at City University London, UK. His main research interests include wireless ad hoc networks, quality of service in wireless networks, and network reliability. He published over 40 papers in international journals and conferences and has been a member of the TPC for a number of IEEE conferences, including Globecom, MASS and WCNC. He holds a Ph.D. in Telecommunications from the University of London, UK and a Dipl-Ing degree in Electronic Engineering from the University of Belgrade, Serbia.

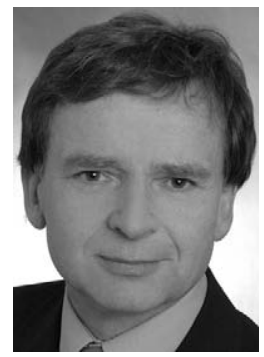

Joachim Habermann (Senior Member IEEE) received the MSc. and Ph.D. degrees in Electrical Engineering from Darmstadt Technical University, Germany in 1979 and 1984. In 1984 he joined ABB research centre in Baden, Switzerland, where he worked in the Telecommunications department in the area of mobile communications. Since 1990 he is Professor at the University of Applied Sciences Giessen-Friedberg/Germany. 